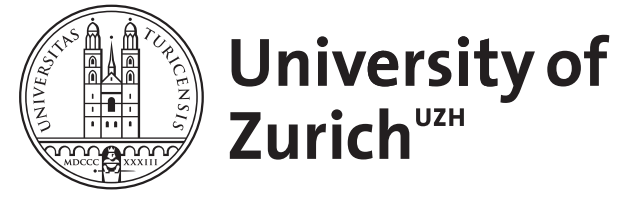

\title{
Schülerprotokolle aus unterschiedlichen Perspektiven lesen - eine explorative Studie
}

Rüede, C ; Weber, C

\begin{abstract}
Mathematiklehrerinnen und -lehrer lesen Schülerprodukte je nach Verwendungszweck aus unterschiedlichen Perspektiven. In dieser Studie wurde mit Repertory Grids erhoben, wie zwölf Lehramtsstudierende und Lehrpersonen Schülerprotokolle lesen und miteinander vergleichen. Aus den Grids wurden ein Kategoriensystem entwickelt und sechs unterschiedliche Perspektiven rekonstruiert. Sie erfassen, dass die Schülerprotokolle entweder beschrieben oder gewertet wurden und sich die Lektüre auf formale, inhaltliche oder personale Aspekte der Texte richtete. Damit sind Lehrpersonen in der Lage, Schülertexte aus vielfältigen Perspektiven zu lesen, besonders wenn sie dazu angehalten werden. Dies ist bedeutsam, weil ein schülerorientierter, differenzierender Unterricht eine Vielfalt von Perspektiven bei den Lehrenden voraussetzt.
\end{abstract}

DOI: https://doi.org/10.1007/s13138-011-0030-6

Posted at the Zurich Open Repository and Archive, University of Zurich

ZORA URL: https://doi.org/10.5167/uzh-64435

Journal Article

Accepted Version

Originally published at:

Rüede, C; Weber, C (2012). Schülerprotokolle aus unterschiedlichen Perspektiven lesen - eine explorative Studie. Journal für Mathematik-Didaktik, 33(1):1-28.

DOI: https://doi.org/10.1007/s13138-011-0030-6 


\title{
Schülerprotokolle aus unterschiedlichen Perspektiven lesen - eine explorative Studie
}

Christian Rüede, Christof Weber (corresponding author)

Christian Rüede, Pädagogische Hochschule Nordwestschweiz, Baslerstrasse 45, 5201 Brugg, Schweiz

Tel. +41524600613

E-Mail: christian.rueede@fhnw.ch

URL: http://www.fhnw.ch/personen/christian-rueede/

Christof Weber, Pädagogische Hochschule Nordwestschweiz, Kasernenstr. 31, 4410 Liestal, Schweiz

Tel. +416192577 77

Fax +41619257766

E-Mail: christof.weber@fhnw.ch

URL: http://www.fhnw.ch/personen/christof-weber/

Schlüsselwörter Leseperspektiven · Schülerprotokolle · Textverstehen · Repertory Grids · Empirische Lehrerforschung $\cdot$ Sekundarstufe 2

Mathematics Subject Classification (2000) 97B50 $97 C 50 \cdot 97 C 70 \cdot 97 D 70$

\begin{abstract}
Kurzfassung Mathematiklehrerinnen und -lehrer lesen Schülerprodukte je nach Verwendungszweck aus unterschiedlichen Perspektiven. In dieser Studie wurde mit Repertory Grids erhoben, wie zwölf Lehramtsstudierende und Lehrpersonen Schülerprotokolle lesen und miteinander vergleichen. Aus den Grids wurden ein Kategoriensystem entwickelt und sechs unterschiedliche Perspektiven rekonstruiert. Sie erfassen, dass die Schülerprotokolle entweder beschrieben oder gewertet wurden und sich die Lektüre auf formale, inhaltliche oder personale Aspekte der Texte richtete. Damit sind Lehrpersonen in der Lage, Schülertexte aus vielfältigen Perspektiven zu lesen, besonders wenn sie dazu angehalten werden. Dies ist bedeutsam, weil ein schülerorientierter, differenzierender Unterricht eine Vielfalt von Perspektiven bei den Lehrenden voraussetzt.
\end{abstract}

\section{Reading student solutions from different perspectives - an exploratory study}

Mathematics teachers read students' work from different perspectives, depending on the intended purpose.

Repertory grids were used in this study to ascertain how twelve teacher trainees and teachers read and compare records of student solutions. A set of categories was developed and six different perspectives were reconstructed on the basis of the grids. These show that the teachers either described the student solutions or formed a critical opinion on them. They read with a focus on formal, content-related or personal aspects of the texts. Teachers are thus able to read student solutions from a variety of different perspectives, particularly when they are specifically 
asked to do so. This is important, because student-oriented lessons depend on teachers having a variety of perspectives at their disposal.

Keywords Reading perspectives $\cdot$ Student solutions $\cdot$ Understanding texts $\cdot$ Repertory grids $\cdot$ Empirical teacher research · Upper Secondary School 


\section{Einleitung}

Wie lesen Lehrpersonen Aufgabenbearbeitungen von Schülerinnen und Schülern? Worauf schauen sie dabei? Zu diesen Fragen liegen bereits einige mathematik-didaktische Studien vor. Sie gehen in der Regel präskriptiv vor und diskutieren die Fragen vor dem Hintergrund einer vorgegebenen Perspektive. So wird in Erhebungen zum Professionswissen das Wissen über mathematische Schülerkognitionen und -vorstellungen als ein Wissen über Schülerfehler und -schwierigkeiten operationalisiert. Die untersuchten Lehrpersonen müssen dann Misskonzepte aus vorgelegten Schülerprotokollen rekonstruieren - was nichts anderes heißt, als dass sie eine bestimmte Leseperspektive einnehmen müssen (Krauss et al. 2008; Hill et al. 2008). Auch in Studien, die spezifische Aspekte beim Lesen von Schülerprotokollen untersuchen, wie etwa das Kommunizieren und Argumentieren (Drüke-Noe 2009), Lernstrategien (Holzäpfel et al. 2009) oder Modellierungsprozesse (Jordan u. vom Hofe 2008), sind die gewünschten Leseperspektiven bereits durch die Vorgaben der Untersuchung festgelegt.

Wie eine Lehrperson einen Schülertext liest, hängt wesentlich von der Perspektive ab, die sie einnimmt (zum Begriff der Perspektive siehe Abschnitt 2). So kann sie gezielt nach Defiziten suchen. Sie kann sich aber auch für die Besonderheiten oder gar für den Sinn des Texts interessieren, für dessen ,innere Rationalität“ (Prediger 2010, S. 78). Diese beiden sehr unterschiedlichen Perspektiven sind in der Mathematik-Didaktik unter dem Begriff der Defizit- bzw. Kompetenzorientierung (Spiegel u. Selter 2007) geläufig. In der Deutschdidaktik spricht man ganz analog vom Defizitblick bzw. der Könnensperspektive (Spitta 1999) oder vom Suchen nach Defiziten und Qualitäten (Ruf u. Winter 2006). Es ist offensichtlich, dass diese sehr unterschiedlichen Leseperspektiven der Lehrperson zu sehr verschiedenen Folgerungen und Konsequenzen für den weiteren Unterricht führen.

Unseres Wissens gibt es nur wenige Studien, die sich für die Perspektiven interessieren, die Lehrpersonen beim Lesen von Schülertexten tatsächlich einnehmen, ohne bereits vorgegeben worden zu sein. In einer entsprechenden Untersuchung von Maier wurden Lehrpersonen Texte von Schülerinnen und Schülern vorgelegt mit der Bitte, diese ,textlichen Eigenproduktionen“ (Maier 2002) zu interpretieren. Im Ergebnis fiel es den befragten Lehrpersonen unerwartet schwer, die Schülertexte angemessen zu analysieren. Deshalb 
formuliert Maier im Anschluss daran vier Eigenschaften, die Interpretationen von Schülertexten aufweisen müssen: Sie sollen „,deskriptiv“, „,komplementär“, ,,vollständig“ und „offen“ sein (ebd.). Im gleichen Sinne fordern andere Autorinnen und Autoren, Lehrpersonen müssten eine ,interpretative Kompetenz“ erwerben (Jungwirth et al. 2001).

Inwiefern sind Leseperspektiven didaktisch relevant? - Stellt man sich Unterricht unter einem Modell von Angebot und Nutzung vor (Fend 1998; Helmke 2006, 2009), wird der von der Lehrperson gestaltete Unterricht als Angebot und die dadurch initiierten Lernprozesse seitens der Schülerinnen und Schüler als Nutzung des Lehrangebots angesehen (Abb. 1, links). Je besser Angebot und Nutzung aufeinander zugeschnitten sind, desto höher ist die Qualität des Unterrichts einzustufen. Entsprechend führt Helmke, wenn er Merkmale für einen guten Unterricht nennt, nicht nur die Klassenführung oder das Unterrichtsklima an, sondern eben auch die Passung von Angebot und Nutzung. So schreibt er: „Passung ist aus meiner Sicht das Schlüsselmerkmal. Es stellt die Grundlage für Konzepte der Differenzierung und Individualisierung dar.“ (Helmke 2006, S. 45) Dabei seien für die Passung nicht nur die Lehrpersonen und Lernenden verantwortlich, sondern auch die Rahmenbedingungen, unter denen Unterricht stattfindet, also das Bildungssystem, die Ressourcen, das soziale Umfeld usw. Da solche Rahmenbedingungen für unsere Studie eine untergeordnete Rolle spielen, werden sie im Weiteren nicht berücksichtigt.

Das Angebot-Nutzungs-Modell verleitet dazu, einen wesentlichen Aspekt eines schülerorientierten Unterrichts auszublenden. So berichtet Helmke, wie ihn Meyer in einem Interview auf folgende Umkehrung anspricht: „Nicht nur die Lehrer machen Angebote an die Schüler. Auch die Schüler machen Angebote an die Lehrer und unterstützen sie dadurch beim Lehren.“(Helmke 2009, S. 76) In seiner Antwort räumt Helmke dann ein, dass zwischen zwei verschiedenen Angebot-Nutzungs-Prozessen zu unterscheiden sei, nämlich solchen, die von den Lehrpersonen ausgehen, als auch solchen, die von Schülerinnen und Schülern ausgehen. Allerdings führt er nicht weiter aus, was unter letzteren Prozessen zu verstehen ist (Abb. 1, rechts).

Ein Unterrichtskonzept, das beide Prozesse einsetzt, ist das Dialogische Lernen (Ruf u. Gallin 1998). In einem derartigen Unterricht unterbreitet die Lehrperson der Klasse ein Angebot, indem sie ihr eine Aufgabe stellt. Die Schülerinnen und Schüler setzen sich mit dieser Aufgabe auseinander und dokumentieren dies schriftlich. Anschließend geben sie ihre 
Dokumente ab, und zwar im Sinne eines Angebots. Die Lehrperson wiederum nutzt dieses Angebot, indem sie interessante Passagen in den Dokumenten ausmacht und zusammenstellt (Ruf u. Gallin 2003, Band II, S. 244-252). Diese Sammlung von Originalbeiträgen wird für die Gestaltung des anschließenden Unterrichts herangezogen und als Grundlage für den nächsten Lernschritt eingesetzt. Damit erweitert sich der Kreislauf von Angebot und Nutzung (Badr Goetz 2007; Ruf 2008).
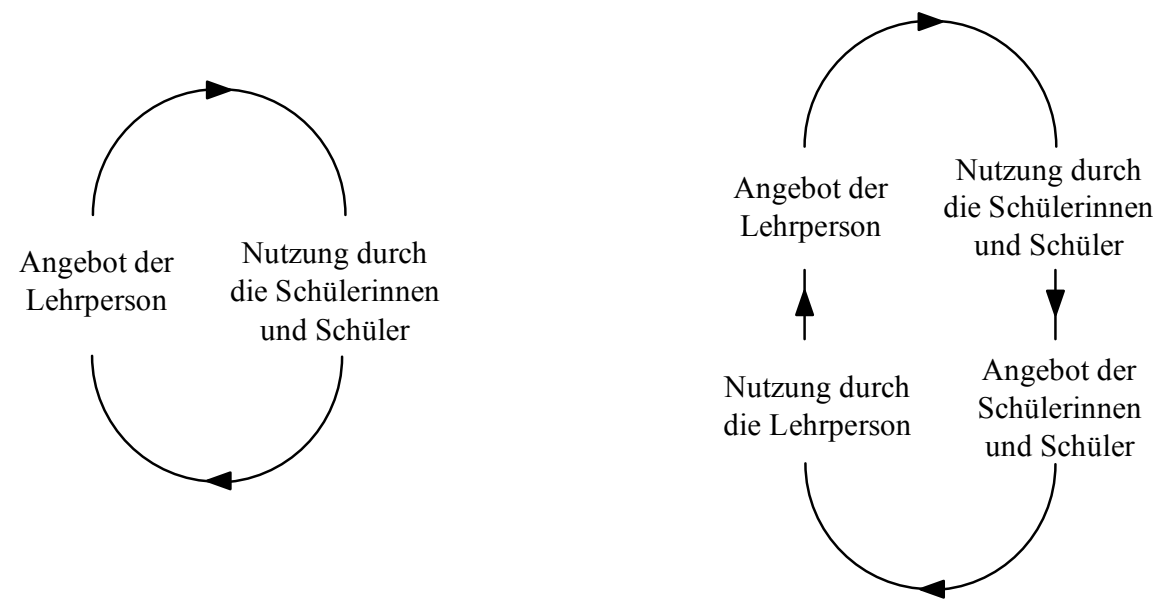

Abb. 1: Das Angebot-Nutzungs-Modell von Unterricht (vereinfacht, nach Fend und Helmke) und seine Erweiterung (nach Badr Goetz und Ruf)

Die folgende Studie ist vor dem Hintergrund eines derart erweiterten Angebot-NutzungsModells (Abb. 1, rechts) zu sehen. Ausgehend vom Schülerangebot, den Schülerprotokollen (zum Begriff siehe Abschnitt 3.1), interessiert hier, wie Lehrpersonen diese Texte lesen und welche Perspektiven (zum Begriff siehe Abschnitt 2) sie bei der Lektüre einnehmen, wenn sie dabei nicht eingeschränkt werden. Damit lautet unsere Forschungsfrage wie folgt:

Welche Perspektiven nehmen Lehrpersonen beim Lesen von Schülerprotokollen ein?

Diese Frage ist deshalb von hoher didaktischer Relevanz, da die eingenommene Leseperspektive darüber mitentscheidet, ob eine Lehrperson nachvollziehen kann, was ihre Schülerinnen und Schüler gedacht haben. Für einen schülerorientierten, differenzierenden Unterricht genügt es gerade nicht, nur die Endergebnisse auf ihre Richtigkeit hin zu überprüfen. Was eine Lehrperson aus den Angeboten ihrer Schülerinnen und Schüler macht und wie sie - darauf aufbauend - die Arbeitsanweisungen für den weiteren Unterricht konstruiert, hängt maßgeblich davon ab, welche Perspektiven sie einnimmt. 
Mit unserer Forschungsfrage wird also das Terrain exploriert und abgesteckt, aus dem heraus die Nutzung von Schülerangeboten erst erwächst (vgl. Abb. 1, rechts). In dieser Studie wird die Vielfalt möglicher Leseperspektiven erhoben und unter die Lupe genommen, die Frage nach der Nutzung von Schülertexten im Unterricht ist nicht ihr Gegenstand.

\section{Der Begriff der Perspektive im Rahmen des Lesens und Verstehens von Texten}

Worauf Lehrpersonen beim Lesen eines Schülerprotokolls schauen und wie sie es tun, verstehen wir als Frage nach der Perspektive, unter der sie das Schülerprotokoll lesen. Deshalb wird im folgenden Abschnitt der Begriff der Perspektive im Kontext von Theorien des Lesens und Textverstehens dargestellt.

Das Lesen von Texten wird im Allgemeinen als konstruktiver und interaktiver Prozess zwischen einem Text und der ihn lesenden Person aufgefasst (für eine detaillierte Darstellung der entsprechenden theoretischen Konzepte und empirischen Befunde siehe Ballstaedt, Mandl, Schnotz u. Tergan 1981; Christmann 2004; Grzesik 1990). Während dieses Prozesses werden Schlüsse aus dem Text gezogen. Wie der Text verstanden wird, manifestiert sich darin, worauf geschlossen wird. Die innerhalb des Texts hergestellten Bezüge entsprechen dabei dem aktuellen Verständnis des Texts und somit der Textinterpretation. Die Textinterpretation hängt aber auch von Randbedingungen ab: „Der Vorgang des Verstehens zieht auch situative, also nichtlinguistische Faktoren mit heran. Und zweitens: Verstehen ist ein zielgerichteter Vorgang.“ (Hörmann 1980, S. 25) So wird immer auch entschieden, welche der grundsätzlich möglichen Schlüsse aus dem Text gezogen werden. Eine solche Entscheidung kann schon vor der Lektüre in Form einer Erwartung vorhanden sein, die an den Text herangetragen wird (Heinemann u. Viehweger 1991, 259 f.), oder aber sie wird erst im Laufe des Lesens aufgrund der Auseinandersetzung mit dem Text getroffen.

In der Kognitionspsychologie spricht man von Perspektiven (,,perspectives“"), wenn es um die Entscheidung geht, welche Elemente eines Texts bei dessen Lektüre bedeutsam werden und welche nicht (Kaakinen u. Hyönä 2005; Pichert u. Anderson 1977; Schraw u. SperlingDennison 1994). So kamen Pichert und Anderson (1977) zum Schluss, dass die Perspektive einer Person darüber entscheidet, was sie für wichtig hält und was für unwichtig. In ihrer Untersuchung legten sie Probanden einen Text über einen Jungen vor, der seinem Freund das 
Haus der Eltern zeigt. Einige Probanden hatten den Text aus der Perspektive eines potentiellen Hauskäufers zu lesen, andere Probanden aus der Perspektive eines möglichen Diebs. Im Anschluss sollten sie - immer noch aus der jeweiligen Perspektive - einzelne Passagen des Texts auf ihre Wichtigkeit hin einschätzen. Neuere Experimente zeigen, dass die eingenommene Perspektive auch darüber entscheidet, an welche Textstellen man sich erinnert und was man einem Text entnimmt (Kaakinen u. Hyönä 2005). Allgemein modelliert die Kognitionspsychologie die Einnahme einer Perspektive als die Aktivierung von Schemata einer hierarchisch hohen Stufe, die die während der Lektüre wirksamen Wissensbestände regulieren (Kaakinen u. Hyönä 2005; Pichert u. Anderson 1977). Mit anderen Worten kann die Perspektive als Intention aufgefasst werden, mit der ein Text gelesen wird, und damit als Ziel, das durch die Lektüre erreicht werden soll. Es ist die Perspektive einer Person, die bestimmt, was sie bei der Lektüre eines Texts interessant und wichtig findet.

Im Rahmen unserer explorativen Studie schließen wir deshalb von dem, was einer Lehrperson bei der Lektüre auffällt oder was sie als wichtig erachtet, auf die Perspektive zurück, die sie eingenommen hat. Ohne auf die Ergebnisse (siehe Abschnitt 5) vorzugreifen, seien hier bereits einige Beispiele von Leseperspektiven genannt. So können Schülerprotokolle etwa daraufhin befragt werden, welche Grundvorstellungen in ihnen sichtbar werden (Hafner u. vom Hofe 2008). Schülerprotokolle können aber auch dahingehend gelesen werden, ob und wie sie sich zur Unterrichtsfortsetzung nutzen lassen (Ruf u. Gallin 2005), welche kognitiven Strukturen sich in ihnen erkennen lassen (Kaune 2007), ob fachliche Normen erfüllt sind (Helmke 2009) oder - sehr viel bereichsspezifischer - wie rationale Zahlen dargestellt sind (Moskal u. Magone 2000).

Wenn es wie in der vorliegenden Studie darum geht, die Vielfalt von Perspektiven zu erheben, dürfen keine Vorgaben gemacht werden, unter welcher Perspektive die Texte zu lesen sind. Zu welcher Methode uns dies geführt hat, wird im folgenden Abschnitt beschrieben. Die eigentliche Untersuchung und ihre Durchführung werden im Abschnitt 4 vorgestellt.

\section{$3 \quad$ Methode}

Unsere Untersuchung verfolgt den Anspruch, realitätsnah und praxisrelevant zu sein. Sie soll ihren Ausgang in der Unterrichtsrealität nehmen, damit eine Theorie entwickelt werden kann und nicht, damit eine Theorie an der Praxis überprüft wird. Praxisrelevanz bedeutet auch, dass 
die Versuchspersonen nicht nur an einer Untersuchung teilnehmen, sondern darüber hinaus für die eigene Unterrichtspraxis profitieren. Da es mit unserer Forschungsfrage darum geht, neue theoretische Konzeptionierungen zu gewinnen, soll ihre Sprache möglichst unvoreingenommen erfasst werden. Auf diese Weise versprechen wir uns, zu didaktisch bedeutsamen und gleichzeitig praxisrelevanten Perspektiven zu kommen. Dabei sind wir uns der Grenzen unseres Anspruchs bewusst, ist doch jede Interpretation der Sprache von Personen prinzipiell nicht frei von den Wertungen ihrer Interpreten. (Für unseren Umgang damit siehe Abschnitt 3.2.)

Methodisch haben wir diesen Anspruch umzusetzen versucht, indem wir Lehramtsstudierenden und Lehrpersonen im Rahmen einer universitären Aus- bzw. Weiterbildungsveranstaltung reale Schülertexte vorlegten, die bei der Bearbeitung offener Aufträge (Genaueres siehe Abschnitt 3.1) entstanden waren. Zur Erhebung der subjektiven Einschätzungen wurden die Versuchspersonen dazu angehalten, denselben Text unter verschiedenen Perspektiven zu lesen und ihre so vorgenommenen Einschätzungen in eigenen Worten wiederzugeben (Repertory Grids, siehe Abschnitt 3.2). Anschließend wurden aus den genannten Begriffen induktiv Kategorien (Abschnitt 3.3) gebildet, um daraus die gesuchten Perspektiven zu rekonstruieren.

\subsection{Offene Aufträge und Schülerprotokolle zur Aktivierung von Einschätzungen}

Eine mathematische Aufgabe, die möglichst vielfältige Bearbeitungs- und Lösungswege wahrscheinlich macht und darüber hinaus starke wie schwache Lernende gleichermaßen herausfordert, ist ein offener Auftrag (Ruf u. Gallin 1998). Dieses Instrument zielt ganz dezidiert auf die individuelle Auseinandersetzung aller Schülerinnen und Schüler mit einer mathematischen Frage. Stärker als in Aufgaben, in denen es primär um das Auffinden einer Lösung geht, regen offene Aufträge das Konstruieren und Erfinden neuen Wissens an. Dieser Prozess wird, auch wenn er nicht zu einer Lösung führt, von den Lernenden in ihrer eigenen Sprache festgehalten und schriftlich dokumentiert, zum Beispiel in einem individuellen Reisetagebuch (ebd.) oder Forschungsheft (Hußmann 2003).

In Anlehnung an Selter (1994) werden derartige Texte je nach Darstellungsform textliche (Maier u. Schweiger 1999) oder raumgeometrische Eigenproduktionen (Wollring 1998) genannt. Wir sprechen im Folgenden von Schülerprotokollen, um auszudrücken, dass 
Schülerinnen und Schüler ihr Vorgehen bei der Bearbeitung eines mathematischen Problems mehr oder weniger explizit protokollieren. Solche Dokumente können wie klassische Aufgabenlösungen aussehen, sie können aber neben formalen Berechnungen auch ausführliche Prosatexte und Skizzen enthalten. Gerade weil in ihnen verschiedene, aber nicht ohne weiteres erfolgreiche Lösungsversuche dokumentiert und reflektiert werden, gewähren sie Einblicke in die vielfältigen Denkwege von Lernenden und eignen sich für unsere Untersuchung der Vielfalt möglicher Leseperspektiven. Darüber hinaus handelt es sich bei Schülerprotokollen auch um Texte, wie sie im Berufsalltag der Lehrpersonen angetroffen werden.

\subsection{Repertory Grids zur Erhebung von Einschätzungen}

Die Methode der Repertory Grids wurde in der Mitte des zwanzigsten Jahrhunderts entwickelt, um in der therapeutischen Diagnostik zu erfassen, wie eine Person ihre aktuellen Erlebnisse und Beziehungen einschätzt (sog. Persönlichkeitskonstrukte). Ihr Erfinder, der Psychologe George Kelly, gilt als einer der Wegbereiter des Konstruktivismus (Kelly 1955; Fransella et al. 1977; Scheer 1992).

In der Mathematik-Didaktik wurden Repertory Grids bisher dazu eingesetzt, um zu erheben, welche subjektiven Theorien Personen über Mathematik als Fach, über das Lernen von Mathematik oder über das Beurteilen von Aufgaben aufgebaut haben (Williams u. Pack 1997; Hiskonen 1999; Bruder et al. 2003). In der vorliegenden Untersuchung werden Repertory Grids eingesetzt, um zu erfassen, wie Lehramtstudierende Schülerprotokolle einschätzen. Damit unterscheidet sich unsere Verwendung vom bisherigen Einsatz vor allem im Gegenstand, auf den Repertory Grids angewendet werden. Aber auch in Durchführung und Auswertung der Befragung gibt es Unterschiede (siehe Abschnitte 4.3 und 3.3).

Ganz grundsätzlich sieht die Arbeit mit Repertory Grids so aus, dass eine Person aufgefordert wird, ihre Einschätzungen eines Sachverhalts in einige selbst gewählte, gegensätzliche Begriffspaare zu fassen und so ihre subjektiven Theorien möglichst adäquat und genau wiederzugeben. Diese Begriffe werden in eine zweidimensionale Matrix eingetragen, das eigentliche Grid (ein Beispiel zeigt Abb. 3). Damit gibt diese Methode einerseits klare Regeln zum Ausfüllen der Grids vor, andererseits wird die befragte Person sprachlich nicht gelenkt oder eingeschränkt. 
Die Regeln zum Ausfüllen von Repertory Grids sind für Lehrpersonen einfach verständlich, da sie teilweise vergleichbare Anforderungen stellen wie der Umgang mit Schülertexten im Berufsalltag. So müssen hier wie dort Aufgabenbearbeitungen von Lernenden gelesen werden, und hier wie dort müssen diese Denkwege miteinander verglichen und eingeschätzt werden. Es gibt allerdings auch Unterschiede. So werden die Lehrpersonen durch Repertory Grids angehalten, dieselben Aufgabenbearbeitungen mehrfach und unterschiedlich einzuschätzen, während im Berufsalltag die Aufgabenbearbeitungen einer Klasse meistens nur auf ihre Richtigkeit überprüft werden. Damit verlangsamen Repertory Grids den Umgang mit den Texten beträchtlich. Er kann sich durchaus auch intensivieren, etwa wenn interessante Passagen in den Aufgabenbearbeitungen entdeckt werden, die sonst übersehen worden wären.

Da die Befragten nur einige wenige Begriffe selbst formulieren, fallen ihre Einschätzungen in der Regel ziemlich pointiert aus. Es ist für die Auswertung von nicht zu unterschätzender Bedeutung, dass jedes ausgefüllte Grid nur einen kleinen, überschaubaren Textkorpus darstellt. Während Repertory Grids in der klassisch-therapeutischen Verwendung als Grundlage für ein Gespräch dienen, werden sie in unserer Studie nur zu Erhebungszwecken eingesetzt, das heißt, bei ihrer Auswertung ist die Versuchsperson nicht zugegen. Um die Begriffe möglichst im Sinne der befragten Person zu interpretieren, sind deshalb die Wahl der Begriffe und die Einschätzungen schriftlich zu begründen. Aber selbst dann sind Missverständnisse und Fehlinterpretationen nicht auszuschließen (siehe Abschnitt 3.3).

\subsection{Qualitative Inhaltsanalyse zur Auswertung der Einschätzungen}

Die gewählten Begriffe in den Grids - die Einschätzungen von Schülerprotokollen - sind sprachliche Eigenproduktionen. Für die Verwendung und Auswertung dieser empirischen Daten werden je nach Zweck und Datenlage sehr unterschiedliche Methoden eingesetzt. So dienen Grids im therapeutischen Gebrauch als Grundlage für ein Gespräch mit einer Person. Im gleichen Kontext werden Grids auch interpersonell miteinander verglichen. Zur Datenanalyse können ausgefüllte Grids aber auch quantitativen Verfahren (Faktoren- oder Hauptkomponentenanalysen) unterworfen werden. Falls sich einige wenige Hauptkomponenten ergeben, können sie als die wesentlichen Konstrukte einer Person oder einer Gruppe von Personen angenommen werden (Scheer 1992; Fischler 2000). Kleinere Grids lassen sich mit der formalen Begriffsanalyse auswerten und in einem Liniendiagramm visualisieren (Spangenberg u. Wolff 1993; Bruder et al. 2003). 
Für unser Ziel - die Benennung von Perspektiven und die Erfassung ihrer Vielfalt aus textanalytischer Sicht - bietet es sich an, die Daten einer qualitativen Inhaltsanalyse zu unterzuziehen. Bei dieser sozialwissenschaftlichen Methode werden aus dem sprachlichen Datenmaterial einige wenige Kategorien abgeleitet, unter die möglichst viele der Daten subsumiert werden können (Atteslander 2010). Gerade wenn es wie bei uns darum geht, möglichst nah am erhobenen Datenmaterial zu bleiben, empfehlen Autoren wie Mayring, sich nicht auf vorab formulierte theoretische Konzepte zu beziehen, sondern die Kategorien induktiv, das heißt direkt aus dem empirischen Datenmaterial heraus abzuleiten (Mayring 2008a, 74 ff.; Mayring 2008b, 11 ff.). Unter der Annahme, dass von den Begriffen in den Grids auf die Leseperspektiven einer Person geschlossen werden kann, lassen sich aus den gebildeten Kategorien die ersten Elemente einer Theorie bestimmen und die gesuchten Perspektiven rekonstruieren.

Natürlich lassen sich bei einer induktiven Theoriebildung weder die Fragestellung und der theoretische Hintergrund der Untersuchung noch das theoretische Wissen der Forscher restlos ausblenden. Diese und weitere forschungsmethodische Kritik an der qualitativen Inhaltsanalyse ist seit ihrer Präsentation immer wieder vorgebracht worden und kann hier nicht diskutiert werden (für eine Diskussion siehe z. B. Thomas u. James 2006). Um möglichst reliable und gleichzeitig valide theoretische Konzeptionierungen zu erhalten, setzen wir die gebildeten Kategorien der kommunikativen Validierung aus (Mayring 2008a, 110 ff.). Was das in unserem Fall heißt, wird in Abschnitt 4.3 ausgeführt. Insgesamt wird also „weicher“ (Mayring 2008b, S. 13) vorgegangen als etwa in einer quantitativen Inhaltsanalyse.

\section{$4 \quad$ Durchführung}

Da die Daten im Rahmen einer Lehrveranstaltung für Lehramtsstudierende (Sekundarstufe 2) erhoben wurden, stellt die Lehrveranstaltung eine Randbedingung der Untersuchung dar und muss hier kurz beschrieben werden. Der Schwerpunkt dieses Abschnitts liegt jedoch darauf, die Erhebung und die Auswertung der Daten vorzustellen und zu illustrieren.

\subsection{Lehrveranstaltung „Wege mathematischen Denkens im Unterricht“}

In den Frühlingssemestern 2008 und 2009 führten wir (Christian Rüede, Christof Weber) an der Eidgenössisch-Technischen Hochschule und an der Universität Zürich die Lehrveranstaltung „Wege mathematischen Denkens im Unterricht“ durch. Sie fand im Rahmen der mathematik-didaktischen Lehramtsausbildung statt, die mit dem „Master of 
Advanced Studies in Secondary and Higher Education“ (Lehrbefähigung Sekundarstufe 2) abgeschlossen wird, und gehörte in den Wahlpflichtbereich.

In der Vorlesung wurden sechs Theorien aus der (mathematik-)didaktischen Forschung vorgestellt, die unterschiedliche Aspekte mathematischen Denkens und Könnens erfassen: prädikative und funktionale Präferenzen (Schwank 1996, 2003; Kaune 2007), Konzepte von Experten und Novizen (Chi et al. 1981), Grundvorstellungen mathematischer Inhalte (vom Hofe 1995, 2003), visueller und analytischer Denkstil (Borromeo Ferri 2004), Semiotik (Fischer 2006; Dörfler 2008) sowie Typen mathematischer Fähigkeiten (Krutetskii 1976; Dahlke 1978). Alle Theorien wurden von den Studierenden und von uns anhand von Originalarbeiten vorgetragen und diskutiert.

Um zu einer vertieften Auseinandersetzung mit den diskutierten Theorien anzuregen, erhielten die Studierenden - unsere Versuchspersonen (siehe Abschnitt 4.2) - dreimal ein Set von Schülerprotokollen (Abschnitt 4.3). Diese Dokumente mussten - als Studienleistung gelesen und mit Hilfe von Repertory Grids eingeschätzt werden, ohne dass die Vorlesung bereits auf die Schülerdokumente Bezug genommen hatte. Dadurch sollten die Studierenden die mathematik-didaktischen Theorien für sich und ihren Schulalltag bedeutsam machen. Gleichzeitig erhielten wir die gewünschten Daten für unsere Untersuchung. Im Anschluss an die Lehrveranstaltung entwickelten wir daraus das Kategoriensystem und rekonstruierten dann die eingenommenen Perspektiven (Abschnitt 4.4).

\subsection{Versuchspersonen}

An den beiden Lehrveranstaltungen - und damit auch an unserer Studie - nahmen insgesamt zwölf Personen ( 7 m, $5 \mathrm{w}$ ) teil. Acht Studierende ( $5 \mathrm{~m}, 3 \mathrm{w})$ absolvierten die Ausbildung zur Gymnasiallehrperson in Mathematik. Entsprechend verfügten sie über keine oder nur wenig Unterrichtserfahrung. Die anderen vier Studierenden (2 m, $2 \mathrm{w})$ waren erfahrene Lehrpersonen, die zum Zeitpunkt der Untersuchung seit mehr als zehn Jahren Mathematik an Gymnasien in Zürich und Umgebung unterrichteten. Sie besuchten unsere Veranstaltung, um sich weiterzubilden.

Alle Versuchspersonen besaßen einen mathematisch-fachwissenschaftlichen Abschluss (Diplom, äquivalent zum Master), weil diese Qualifikation in der Schweiz für Gymnasiallehrpersonen vorausgesetzt wird. Aufgrund der kleinen Stichprobe muss darauf 
verzichtet werden, die Einschätzungen von Untergruppen unserer Versuchspersonen einander gegenüberzustellen (zum Beispiel Frauen gegenüber Männern oder beruflich erfahrene Personen gegenüber beruflich unerfahrenen Personen).

\subsection{Schülerprotokolle und Repertory Grids}

Die Studierenden erhielten dreimal - zu Beginn, in der Mitte und gegen Ende des Semesters ein Set von Schülerprotokollen, zusammen mit dem offenen Auftrag, den die Schülerinnen und Schüler bearbeitet hatten. Die Aufträge wie auch die Schülertexte stammen aus dem gymnasialen Mathematikunterricht (Klassenstufen 10 und 11). Im ersten Auftrag (Abb. 2) musste die Folge von pentagonalen Punktmustern fortgesetzt und algebraisch modelliert werden, ohne dass die Klasse bereits mit dem Thema von Folgen und ihren Bildungsgesetzen bekannt gewesen wäre (für die entsprechende Schulbuchaufgabe siehe Deller, Gebauer u. Zinn 2000, S. 98). Der zweite Auftrag drehte sich um die Erfindung des Skalarprodukts in der analytischen Geometrie, während es im dritten Auftrag darum ging, die Eigenschaften des Logarithmus anhand dessen Funktionsgraphen zu untersuchen.

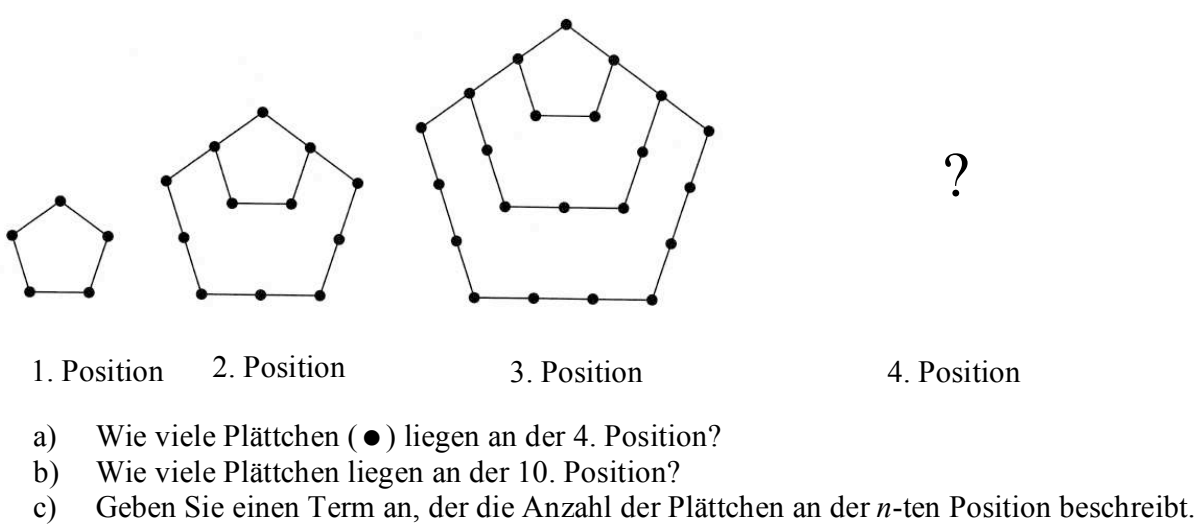

Abb. 2: Offener Auftrag an die Schülerinnen und Schüler

Zusammen mit dem jeweiligen Auftrag erhielten die Studierenden ein Set von sechs bis sieben Schülerprotokollen, das über die ganze Untersuchung hinweg von Lernenden derselben Klasse stammte. Jeder Schülertext umfasst zwischen einer und acht DIN-A4-Seiten und wurde als repräsentativ für den jeweiligen Klassensatz der Aufgabenbearbeitungen herausgegriffen. (Die Arbeitsaufträge an die Studierenden mit den drei offenen Aufträgen und den ausgewählten Schülerprotokollen sind online einsehbar (Rüede u. Weber 2009).) 
Jedes Set von Schülerprotokollen war von den Studierenden nach der Methode der Repertory Grids zu analysieren. Ihre Hausaufgabe bestand darin, sich nach einer ersten Lektüre in einem ersten Schritt zwei Schülertexte herauszugreifen, die sich nach ihrer Einschätzung stark voneinander unterscheiden. Für diese beiden Texte waren zwei eigene, möglichst gegensätzliche Begriffe (sogenannte Merkmale) zu formulieren, die erfassen, inwiefern sich die Texte unterscheiden (z. B. „,erklärend, Gedanken formulierend“ vs. „reines Resultat“, vgl. Abb. 3). Im zweiten Schritt mussten alle Schülertexte daraufhin eingeschätzt werden, wie ausgeprägt die beiden formulierten Merkmale für sie zutreffen, und zwar auf einer dreistufigen Skala $(0,+,++)$. Merkmale wie Einschätzungen wurden von den Studierenden in die ersten beiden Zeilen eines Grids eingetragen (vgl. Abb. 3).

Damit waren eine erste Einschätzung und ein erstes Begriffspaar gewonnen. Um die Studierenden anzuregen, dieselben Schülerdokumente auch unter anderen Perspektiven zu lesen, war der Prozess der Auswahl zweier sich stark unterscheidender Texte, der Formulierung eines gegensätzlichen Merkmalpaars und der Einschätzung aller Schülertexte bis zu fünfmal durchzuführen. Auf diese Weise füllten sie das Grid Zeile für Zeile aus. Es enthielt nun zweierlei: erstens mehrere selbst gewählte Merkmale und damit Kriterien, nach denen die Schülerprotokolle eingeschätzt wurden, und zweitens die Einschätzungen mehrerer Schülerprotokolle hinsichtlich eigener Kriterien.

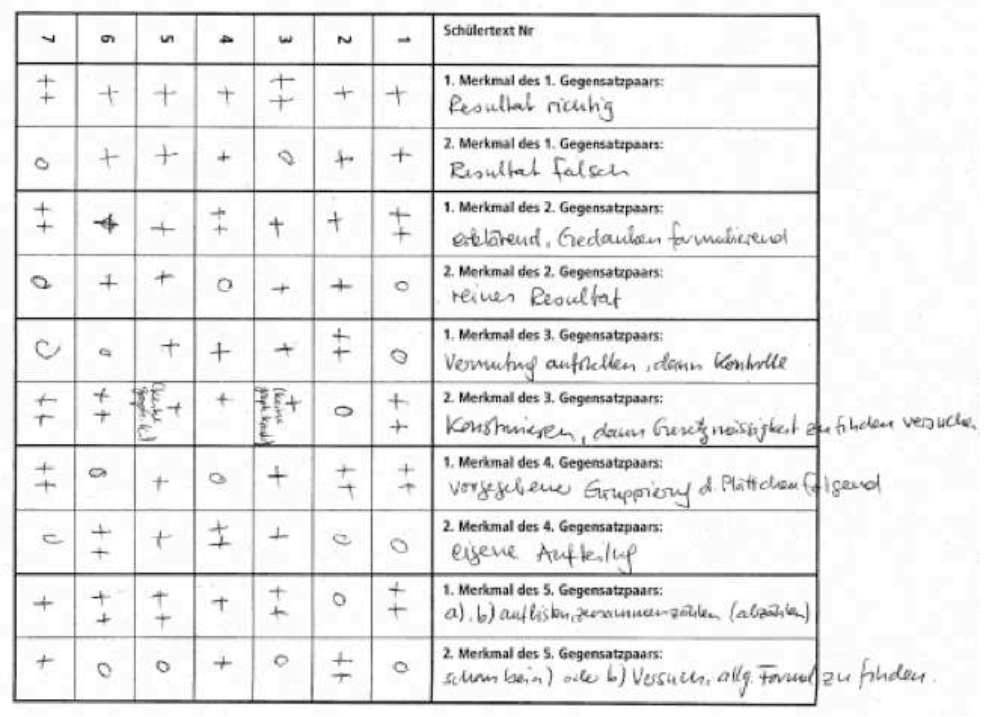

Abb. 3: Ausgefülltes Repertory Grid von Nicole Müller (um $-90^{\circ}$ verdreht abgebildet) ((An den Setzer: Druckvorlage liegt bei!)) 
Zur Illustration zeigt Abbildung 3 das Grid der ersten Analyse von Nicole Müller (Name geändert). Die Studentin hat sieben Schülerprotokolle, die in Folge des in Abbildung 2 gezeigten Auftrags entstanden, auf fünf verschiedene Arten eingeschätzt. Ihr erster Blick gilt der fachlichen Überprüfung des Resultats und führt zum Gegensatzpaar „Resultat richtig“ bzw. „Resultat falsch“. Zwei Texten attestiert sie, dass deren Resultat richtig sei, den andern, dass sie teilweise richtig gelöst seien. Da sie beim Merkmal ,Resultat falsch“ keins der Schülerdokumente mit „,++“ einschätzt, lässt sich nicht erkennen, welche beiden Schülertexte zur Wahl dieses Gegensatzpaars geführt haben könnten.

Jedem ausgefüllten Grid war eine schriftliche Begründung zur Wahl der Gegensatzpaare beizulegen (max. zwei Seiten). Leider gibt der entsprechende Text von Nicole Müller keinerlei Aufschluss darüber, welche beiden Schülertexte zur Wahl ihres ersten Gegensatzpaars geführt haben. Sie schreibt:

„1. Gegensatzpaar ,Resultat richtig“ - ,Resultat falsch“: Es ist mir bewusst, dass dieses Merkmal gerade nicht auf die Art und Weise des Denkvorgangs abzielt. Aber ehrlicherweise spielt die Lösung eine erhebliche Rolle. Beim Bewerten der Texte merke ich, dass es gar nicht so einfach ist. Welche Punkte verteile ich, wenn ich sehe, die Schülerin hat ein Prinzip begriffen, macht aber ganz am Anfang einen Fehler, der sich fortpflanzt?“"

Die Studentin verteidigt hier ihren Anspruch, die Ergebnisse auf ihre Korrektheit hin zu untersuchen und damit gewisse (fachliche) Normen anzulegen zu wollen. Dies könnte auf einen gewissen Erwartungsdruck seitens der Lehrveranstaltung (siehe ihren Titel in Abschnitt 4.1) und damit der Autoren der vorliegenden Studie hinweisen. Wie ihre Begründung nahelegt, macht sie zwar bei einigen Schülertexten falsche Lösungen aus, um dann dennoch auch „richtige“ Überlegungen zu finden. Deshalb vergibt sie beim Merkmal „Resultat falsch“ für keinen einzigen Schülertext die zustimmende Einschätzung „,++“(vgl. Abb. 3).

Erst ab ihrer dritten Analyse formuliert die Studentin Paare von Merkmalen, die beide auf mindestens einen der Schülertexte zutreffen. So untersucht sie in ihrer vierten Analyse, wie sehr die Schülertexte den vorgegebenen Mustern und Strukturen der Aufgabe folgen bzw. wie sehr in ihnen eigene Wege beschritten werden:

„4. Gegensatzpaar ,gegebener Gruppierung folgend‘ - ,eigene Aufteilung‘: Es interessierte mich zu sehen, wer folgt der Konstruktion der angedeuteten Gruppierung und 
wer ,wagt‘ es, eigene Wege zu definieren und zu beschreiten. Dabei liegt die Spannung darin, dass die Andeutungen in solchen Aufgaben oft eine Hilfe bedeuten, jedoch für mathematisches Denken eine gewisse Unkonventionalität und Mut, Dinge anderes als gewohnt zu betrachten, sehr hilfreich sein kann.“

Ruft man sich in Erinnerung, dass dieses Repertory Grid ganz am Anfang des Semesters entstanden ist, fragt man sich unwillkürlich: Macht sich die Studentin mit diesen Zeilen nicht auch selbst „Mut“, „eigene Wege zu beschreiten“ und Schülertexte „,anders als gewohnt“ zu betrachten? Wenigstens kann dies - wie beim Problemlösen - auch bei der Einschätzung von Schülertexten „sehr hilfreich“ sein.

\subsection{Auswertung der Repertory Grids und Bildung von Kategorien}

Da die Bearbeitung der Repertory Grids als Studienleistung eingefordert wurde, haben alle zwölf Studierenden je drei Grids ausgefüllt, ergänzt durch schriftliche Begründungen. Damit lagen am Ende des Semesters sehr unterschiedliche Daten vor: erstens die individuell formulierten Gegensatzpaare von Merkmalen, zweitens die Einschätzungen der Schülerprotokolle hinsichtlich der Merkmale sowie drittens die Begründungen der Gegensatzpaare und Einschätzungen.

Die Aufgabe, die vorgelegten Schülerprotokolle hinsichtlich der Merkmale einzuschätzen, war in erster Linie zur Ausbildung der Studierenden gedacht. Für unsere Untersuchung werden die dabei entstandenen Daten deshalb nicht weiter verwendet. Es werden also nur die von den Versuchspersonen formulierten Merkmale kategorisiert, wobei die entsprechenden Begründungen zum Verständnis der Merkmale herangezogen werden. Eine erste Sichtung der Grids lieferte eine unerwartete Breite der Gegensätzlichkeit in den Merkmalpaaren. Was als gegensätzlich aufgefasst wird, ist immer subjektiv. So nennt eine Studentin das Merkmalpaar „Farben verwendet - viel gestrichen“, was nicht ohne weiteres verständlich ist. Aus diesem Grund werden alle genannten Merkmale nicht wie ursprünglich geplant in Paaren, sondern für sich genommen kategorisiert.

Unser induktives Vorgehen bei der Auswertung der Daten wird nun exemplarisch an Nicole Müllers Repertory Grid (Abb. 3) illustriert. Zur Interpretation ihrer Merkmale wird auch der Begründungstext herangezogen. Das Grid dieser Studentin ist zu diesem Zweck besonders geeignet, gibt es doch eine breite Palette von Merkmalen wieder (ob dies damit 
zusammenhängt, dass das Grid zu Beginn der Lehrveranstaltung entstanden ist, kann nicht entschieden werden):

1. Merkmal „Resultat richtig“ und 2. Merkmal „Resultat falsch“:

Mit ihren ersten beiden Merkmalen wertet Nicole Müller die Korrektheit der Schülerlösungen. Damit trägt sie fachlich-mathematische Kriterien an die Problemlöseprotokolle heran. Mit dem ausschließlichen Fokus auf die Resultate stellt sich ihr das Dilemma, dass der Lösungsweg trotz falscher Lösung weitgehend richtig sein kann. Entsprechend fragt sie sich im Begleittext: ,, [...] Welche Punkte verteile ich, wenn ich sehe, die Schülerin hat ein Prinzip begriffen, macht aber ganz am Anfang einen Fehler, der sich fortpflanzt? " Um nicht nur die formale Korrektheit der Schülerlösungen beurteilen zu können, muss sie diese Frage vorab klären.

- 3. Merkmal ,erklärend, Gedanken formulierend“ und 4. Merkmal „,reines Resultat“: Eine andere Perspektive nimmt die Studentin für ihre beiden nächsten Merkmale ein. Hier wertet sie die Schülerprotokolle nicht, sondern beschreibt, ob die Schülerinnen und Schüler in ihrer Herleitung ihre Gedanken dokumentieren oder nicht. Sie begründet ihre Wahl mit folgender Beobachtung: ,,Einige Schüler beschreiben verbal, was für Gedanken ihnen durch den Kopf gehen, andere schreiben Zahlenreihen hin mit Zwischenschritten, wieder andere gar nichts, sondern bloß das Resultat. [...]“ Wie schon bei den beiden ersten Merkmalen kann sie auch hier auf der Ebene der Darstellung des Schülerprotokolls bleiben. Um abzuklären, ob eine Person ihren Denkweg dokumentiert hat oder nicht, braucht man ihr Protokoll nicht unbedingt inhaltlich zu verstehen. Es genügt, das Dokument auf formale Aspekte hin anzusehen.

- 5. Merkmal „Vermutung aufstellen, dann Kontrolle“ und 6. Merkmal „Konstruieren, dann Gesetzmäßigkeit zu finden versuchen":

Mit ihrem fünften und sechsten Merkmal verlässt Nicole Müller die Darstellungsebene der Texte und richtet ihren Blick auf deren mathematische Inhalte und Bedeutungen. Sie beschreibt zwei unterschiedliche Strategien, wie die Lernenden den mathematischen Auftrag angegangen sind. Ein Schülerprotokoll (Nr. 2) liest sie so, dass darin bereits die wenigen vorgegebenen Zahlenbeispiele genügen, um eine Regel abzuleiten. Andere Schülerdokumente (Nr. 1, 6 und 7) stellt sie in einen Gegensatz dazu, da in ihnen erst viele weitere eigene Beispiele generiert werden, bevor eine Regel vermutet wird. 
7. Merkmal „vorgegebener Gruppierung der Plättchen folgend“ und 8. Merkmal „eigene Aufteilung“:

Mit ihrem siebten und achten Merkmal wagt sich die Nicole Müller noch weiter in ihrer Interpretation der Schülertexte vor. Sie untersucht nun, wer die angedeuteten ringförmigen Zuwächse in den Punktmustern (vgl. Abb. 2) erkennt und sich zunutze macht und wer die Punktmuster auf eine andere, eigene Art gliedert. Dabei interessiert sie sich weniger dafür, wie jemand vorgeht, um das Problem zu lösen, sondern - wie sie selbst schreibt - vielmehr dafür, ob es jemand, ,wagt, eigene Wege zu definieren und zu beschreiten“. Während das fünfte und sechste Merkmal inhaltliche Aspekte erfassen, stellt sich Nicole Müller hier die Frage, warum die Lernenden das mathematische Problem „so“ bearbeitet haben und nicht anders. Sie schließt vom Text auf die Selbständigkeit der Person, von der er stammt.

· 9. Merkmal „a), b) auflisten, zusammenzählen (abzählen)“ und 10. Merkmal „schon bei a) oder b) Versuch, allgemeine Formel zu finden":

Mit ihren beiden letzten Merkmalen schaut die Studentin nochmals auf den Inhalt. Im Gegensatz zu den Merkmalen 5 und 6, bei denen es sich um generelle Vorgehensweisen und Strategien zum Problemlösen handelt, sind diese beiden Merkmale nur im Kontext der vorliegenden Aufgabe sinnvoll: Zählt eine Schülerin bzw. ein Schüler in den Teilaufgaben a) und b) noch einzelne Punkte durch oder versucht sie bzw. er bereits hier, einen Funktionsterm aufzustellen, um die Frage nach der Anzahl Punkte zu beantworten? Während die beiden Merkmale selbst Beschreibungen sind, klingt in ihrer Begründung auch eine gewisse Wertung an. Nicole Müller schreibt: „Hier stehen sich ein gewisser Pragmatismus und Vorausdenken, Kreativität gegenüber. Wo setzt eine Denkleistung ein? Wo ist es reine Fleissarbeit?" Mit solchen Fragen geht sie über die Erklärung und Begründung ihrer Merkmale hinaus.

In Vorbereitung auf die Ergebnisse unserer Studie lassen sich bereits in diesen Interpretationen zwei unterschiedliche „Dimensionen“ erkennen, die verschieden stark ausgeprägt sein können. Sie werden im nächsten Abschnitt zur Bildung der gesuchten Kategorien führen:

1. Alle Merkmale drücken entweder eine wertende (z. B. „Resultat richtig“) oder eine beschreibende Perspektive der Lehramtsstudentin aus (z. B. ,a), b) auflisten, zusammenzählen (abzählen)“). 
2. Merkmale wie „erklärend, Gedanken formulierend“ betreffen die Darstellung eines Schülerprotokolls, während sich andere Merkmale auf den mathematischen Inhalt beziehen. Hingegen fokussieren Merkmale wie „eigene Aufteilung“ auf das Zustandekommen des Protokolls, indem es Aussagen über die Schülerin bzw. den Schüler und z. B. ihre oder seine Selbständigkeit macht. Genau gleich wie oben vorgestellt wurde etwa ein Drittel aller vorliegenden Merkmale von einem der Autoren, dem Erstauswerter (Chr. R.), gelesen und interpretiert, um daraus vorläufige Kategorien (für die er eigene Begriffe verwendet hat) zu bilden. Dazu legte er erste Kategorisierungsregeln fest. So sollte beispielsweise jedes Merkmal genau einer Kategorie zugeordnet werden, oder es sollten nur die Teile des Begründungstexts herangezogen werden, die nicht über die Erklärung und Begründung der Merkmale hinausgingen. Um die Zuverlässigkeit der Zuordnungen zu erhöhen, wählte er für jede Kategorie einige Merkmale aus, die ihm als typisch erschienen. Diese Merkmale dienten im Folgenden als Ankerbeispiele. Anschließend explizierte und begründete der Erstauswerter die gebildeten Kategorien mit den Kategorisierungsregeln und Ankerbeispielen dem anderen Autor gegenüber, dem Zweitauswerter (Chr. W.). Mit diesen Vorgaben wertete der Zweitauswerter dieselben Merkmale noch einmal aus und versuchte, sie unter die Kategorien des Erstauswerters zu subsumieren. Anschließend verglichen die beiden Autoren ihre Zuordnungen der Merkmale. Stimmten sie bei einem Merkmal nicht überein, wurde a) entweder eine bestehende Kategorie überarbeitet (neuer Begriff oder Verfeinerung in Subkategorien), b) das strittige Merkmal einer anderen Kategorie zugeordnet, c) eine neue Kategorie gebildet oder ließ sich d) einer der Auswerter von den Begründungen des anderen überzeugen und rückte von seiner Zuordnung ab. Dabei wurden einige Kategorisierungsregeln überarbeitet oder neu aufgestellt. Auch das eine oder andere Ankerbeispiel musste ersetzt oder neu aufgenommen werden. Als Nächstes wurde der Prozess der induktiven Kategorienbildung auf das ganze Datenmaterial angewendet und so oft durchlaufen, bis ein Kategoriensystem vorlag, mit dem alle unsere Repertory Grids ausgewertet, das heißt jedes Merkmal unter eine Kategorie subsumiert werden konnte.

Wie erwähnt schließen wir von den Merkmalen, die eine Versuchsperson nennt, auf die Perspektiven, die sie bei der Lektüre der Schülerprotokolle eingenommen hat. Folglich konnten mit dem Vorliegen des Kategoriensystems die gesuchten Perspektiven rekonstruiert werden. In der Darstellung der Ergebnisse bezeichnen wir deshalb jede Perspektive mit demselben Begriff wie die ihr zugrunde liegende Kategorie von Merkmalen. 


\section{$5 \quad$ Ergebnisse}

Im ersten Teil dieses Abschnitts wird das Kategoriensystem für die Merkmale unserer Versuchspersonen sowie die daraus rekonstruierten sechs Leseperspektiven vorgestellt. Im zweiten Teil finden sich einige quantitative Ergebnisse unserer Studie.

\subsection{Qualitative Ergebnisse: Kategoriensystem und sechs Perspektiven}

Wie schon bei den diskutierten Beispielmerkmalen in Abschnitt 4.4 lassen sich auch bei allen anderen Merkmalen zwei unterschiedliche „Dimensionen“ ausmachen. Dabei besitzt die erste Dimension zwei, die zweite drei Ausprägungen:

1. Die erste Dimension gibt wieder, ob eine Versuchsperson deskriptiv an einen Schülertext herangeht und ihn so sachlich wie möglich beschreibt oder ob sie normativ an ihn herangeht, das heißt (implizite oder explizite) Normen an ihn anlegt und ihn so wertet. Hier geht es also darum, ob ein Merkmal eine Beschreibung ist oder ob es eine Wertung enthält.

2. Die zweite Dimension gibt wieder, welche Aspekte des Schülertexts ein Merkmal betrifft. Dabei können Merkmale nicht nur die Darstellung (formale Aspekte) oder den mathematischen Inhalt (inhaltliche Aspekte) eines Texts betreffen. Merkmale dieser Dimension können auch Hypothesen darüber sein, wie die Autorin bzw. der Autor des Texts denkt und was sie bzw. er kann. Weil derartige Merkmale einer Person gewisse Eigenschaften zu- oder abschreiben, geben sie personale Aspekte wieder.

Unter den erhobenen Merkmalen kommen alle möglichen Kombinationen zwischen den Ausprägungen der beiden Dimensionen vor. Deshalb lassen sie sich in einer $2 \times 3-$ Kreuztabelle kategorisieren. Das resultierende Kategoriensystem enthält folglich sechs Kategorien (Tabelle 1). Es zeigt zu jeder Kategorie die entsprechenden Subkategorien mit den Ankerbeispielen und benennt die zugehörigen Perspektiven. So lässt sich aus der Tabelle links oben ablesen, dass das Merkmal „Schemata und Zeichen verwendet" formale Aspekte des Texts - genauer: die Art der Darstellung des Texts - beschreibt. Dieses Merkmal lässt auf eine Perspektive der Versuchsperson schließen, die wir formal-beschreibende Perspektive nennen.

\begin{tabular}{|c|c|} 
Merkmal ist & Merkmal ist \\
eine Beschreibung & eine Wertung
\end{tabular}




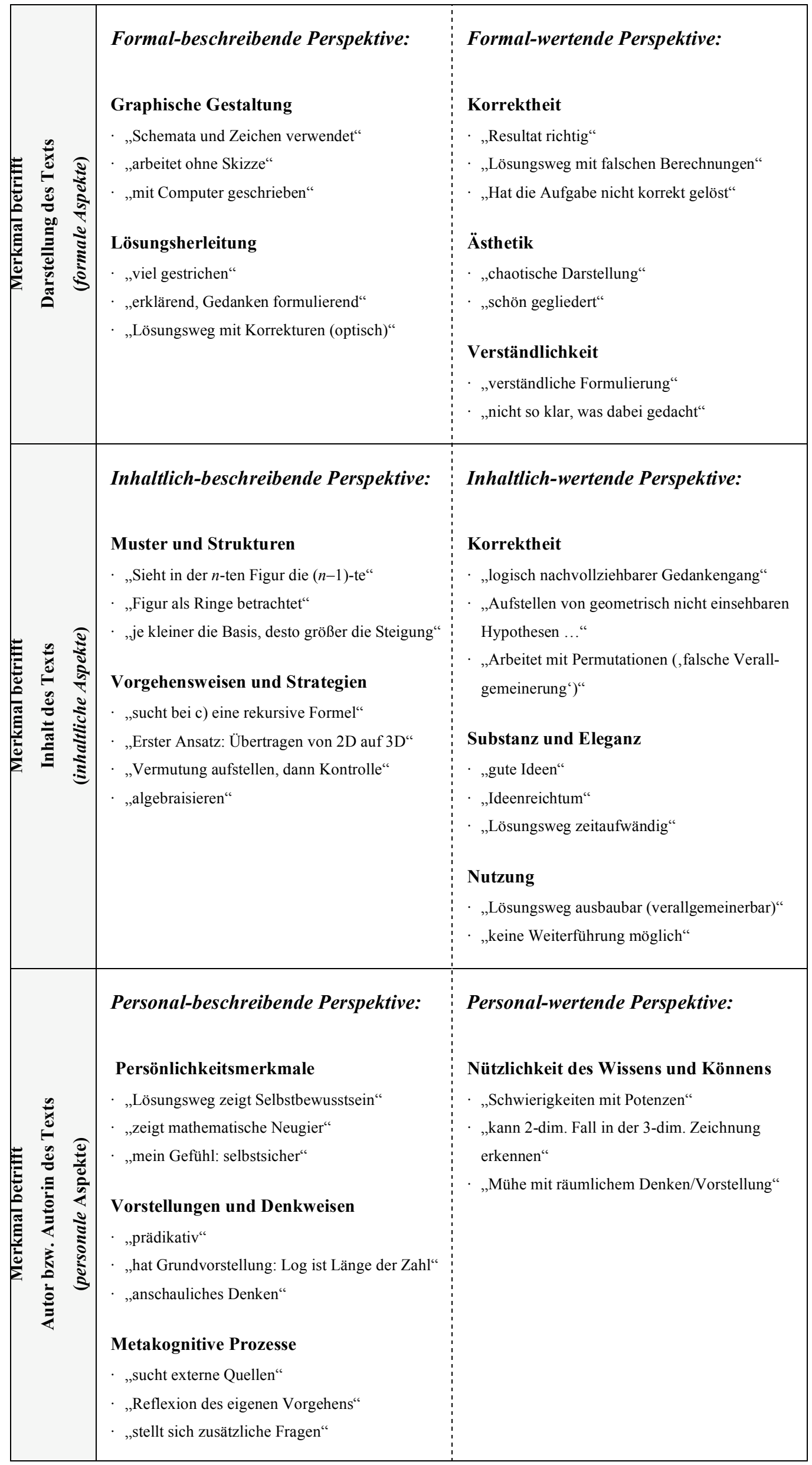




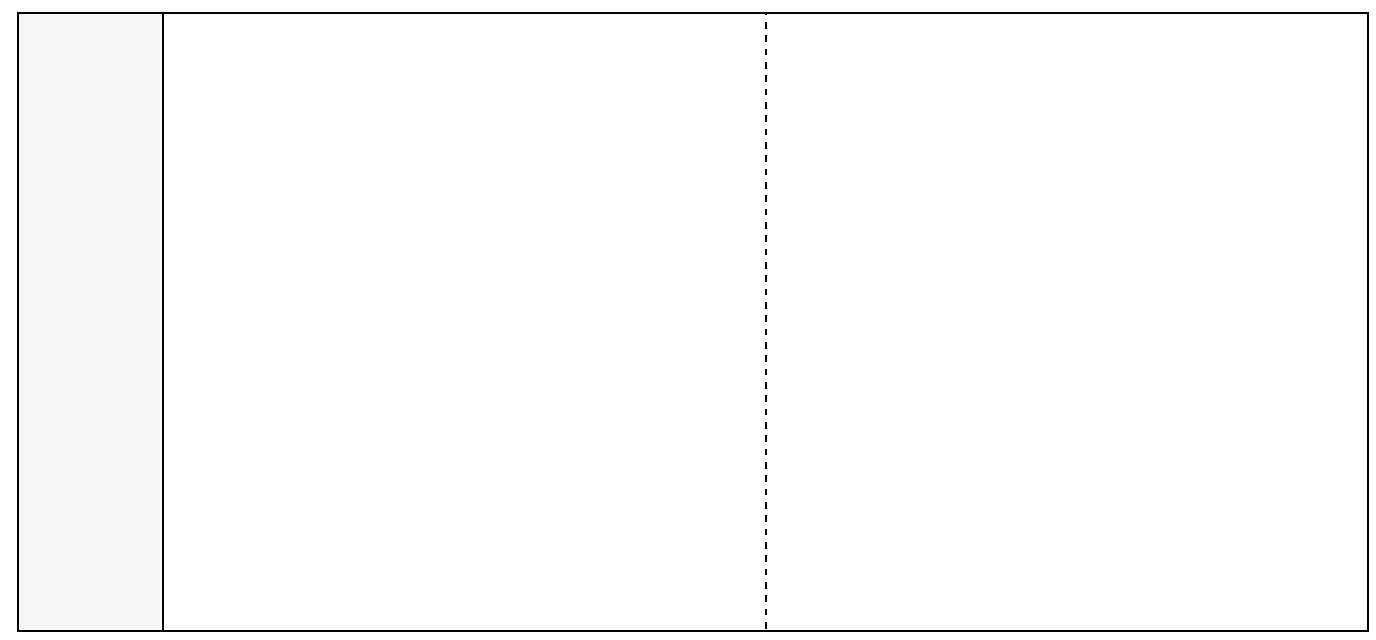

Tab. 1: Kategoriensystem mit Ankerbeispielen und Perspektiven

Die Unterscheidung zwischen Beschreibung und Wertung ist bei der Lektüre von Schülerprotokollen wesentlich (vgl. auch Abschnitt 6.1.1). Sie bescherte uns bei der Kategorisierung der Merkmale einige Schwierigkeiten, da „beschreibend“ und „wertend“ die beiden Pole eines Spektrums sind (deshalb ist die trennende Linie in Tab. 1 durchlässig dargestellt). In den meisten Fällen konnte mit Hilfe der Begründungstexte bestimmt werden, welcher Aspekt eher intendiert ist. In den restlichen Fällen haben wir versucht, über die Einteilung mit geeigneten Kategorisierungsregeln zu entscheiden. So haben wir etwa festgelegt, Persönlichkeitsmerkmale als beschreibend zu kategorisieren, weil wir sie weniger als Bewertung denn als psychologische Eigenschaften einer Person auffassen.

Im Folgenden werden die sechs Perspektiven vorgestellt. In Entsprechung zu den Subkategorien werden typische Fragen formuliert, die zu den genannten Ankerbeispielen der Lehrpersonen geführt haben könnten. Anschließend wird rekonstruiert, welche Perspektiven sie eingenommen haben.

\subsubsection{Formal-beschreibende Perspektive}

Wer unter der formal-beschreibenden Perspektive auf eine Aufgabenbearbeitung schaut, konzentriert sich auf ihre Darstellung und stellt sich folgende Fragen:

Wie wurde der Text graphisch gestaltet und dargestellt?

Wie wurde die Herleitung der Lösung festgehalten?

Die erste Frage zielt auf die Art der graphischen Gestaltung und führte unsere Versuchspersonen zu Aussagen wie „Schemata und Zeichen verwendet“ oder „,mit Computer geschrieben“. Mit der zweiten Frage zielt man auf die Art der Lösungsherleitung, also darauf, 
wie die Schülerinnen und Schüler ihre Überlegungen, die sie zum Ergebnis führten, zu Papier gebracht haben: Wird die Lösungsherleitung ausformuliert, das heißt, drückt sich jemand „erklärend, Gedanken formulierend“ aus? Oder sind Irrwege in der Form von Korrekturen erkennbar, das heißt, wurden Teile des Lösungswegs durchgestrichen? Kurz, unter dieser Perspektive wird die Darstellung eines Schülertexts so sachlich wie möglich beschrieben, ohne ihn zu werten.

\subsubsection{Formal-wertende Perspektive}

Auch aus der formal-wertenden Perspektive interessiert die Darstellung des Schülertexts. Sie wird hier im Gegensatz zur letzten Perspektive jedoch bewertet:

Wurde die Aufgabenbearbeitung korrekt dargestellt?

Wurde die Aufgabenbearbeitung schön dargestellt?

Wurde die Aufgabenbearbeitung verständlich dargestellt?

Hier werden also sehr verschiedene Normen an den Schülertext angelegt. Schaut man auf die Korrektheit, führt das zu Merkmalen wie „Resultat richtig“ oder „hat die Aufgabe nicht korrekt gelöst"“. Dazu muss der Text nicht unbedingt verstanden werden, etwa wenn das Urteil aus dem syntaktischen Vergleich mit einer Musterlösung erfolgt. Geht es um die Ästhetik oder Verständlichkeit der Darstellung, führt dies zu Merkmalen wie „,chaotische Darstellung“ oder „nicht so klar, was dabei gedacht“.

\subsubsection{Inhaltlich-beschreibende Perspektive}

Ganz andere Merkmale entstehen, wenn man nicht auf die Darstellung, sondern auf den mathematischen Inhalt einer Aufgabenbearbeitung fokussiert. Wird ein Schülertext aus einer inhaltlich-beschreibenden Perspektive gelesen, unterwirft man ihn Fragen wie diesen:

Welche Muster und Strukturen der Aufgabe wurden aufgegriffen?

Wie wurde vorgegangen und welche Strategien wurden eingesetzt?

Für die Bearbeitung mathematischer Aufgaben muss man auf Muster und Strukturen zurückgreifen, die in der eigentlichen Aufgabenstellung gegeben sind. Schaut man nun auf die Aufgabenbearbeitung einer Person, kann man fragen, welche dieser Muster und Strukturen sie erkannt und genutzt hat. So kommen unsere Versuchspersonen zu Merkmalen wie „sieht in der $n$-ten Figur die (n-1)-te“ oder „Figur als Ringe betrachtet“. Man kann aber auch nach den Vorgehensweisen und Strategien suchen, die die Person verwendet hat, um die Aufgabe zu bearbeiten. Dies kann dann zu Merkmalen wie „sucht bei c) eine rekursive Formel“ oder „Vermutung aufstellen, dann Kontrolle“ führen. Während Ersteres eine Vorgehensweise 
wiedergibt, die sich auf eine konkrete Aufgabe bezieht, ist das zweite Merkmal eine Strategie, die ganz allgemein zum Lösen von Aufgaben herangezogen werden kann.

\subsubsection{Inhaltlich-wertende Perspektive}

Im Gegensatz zur letzten Perspektive wird der Inhalt gewertet, wenn man aus einer inhaltlichwertenden Perspektive auf den Text schaut und fragt:

- Wurde in der Aufgabenbearbeitung korrekt argumentiert?

- Wurde die Aufgabe schön bearbeitet?

• $\quad$ Lässt sich die Aufgabenbearbeitung ausbauen und weiterführen?

Wie schon bei der formal-wertenden Perspektive geht es auch hier um Korrektheit und Ästhetik, die sich nun aber auf den Inhalt, nicht auf die Darstellung des Texts beziehen. Interessiert die Korrektheit der Aufgabenbearbeitung und damit die des Vorgehens und der eingesetzten Strategien, kann dies zu Merkmalen wie „logisch nachvollziehbarer Gedankengang“ oder „Aufstellen von geometrisch nicht einsehbaren Hypothesen“ führen. Die Frage nach der Substanz und Eleganz einer Aufgabenbearbeitung bezieht sich auf die Reichhaltigkeit und Kürze eines Arguments. Sie kann zu Aussagen wie „Ideenreichtum“ oder „Lösungsweg zeitaufwändig“ führen. Aus einer inhaltlich-wertenden Perspektive heraus kann aber auch nach der Nutzung gefragt werden, also nach dem Potential, das eine Bearbeitung besitzt, um weiter ausgebaut zu werden und zur Lösung führen zu können. Typische Merkmale, die man Schülertexten dann zuschreibt, sind „Lösungsweg ausbaubar (verallgemeinerbar)““ und „keine Weiterführung möglich“.

\subsubsection{Personal-beschreibende Perspektive}

Noch einmal ganz andere Merkmale entstehen, wenn man von einer Aufgabenbearbeitung auf die Person rückschließt, von der sie stammt. Man sucht dann nach Erklärungen, weshalb der Text so und nicht anders aussieht, und damit nach verschiedenen Eigenschaften einer Person, die die Darstellung und den Inhalt ihres Texts bedingen. Nimmt man eine personalbeschreibende Perspektive ein, stellen sich Fragen wie:

- $\quad$ Welche Merkmale und Eigenschaften weist die Schülerin bzw. der Schüler auf?

• $\quad$ Wie denkt die Schülerin bzw. der Schüler?

Welche metakognitiven und selbstreflexiven Prozesse laufen ab?

Die erste Frage interessiert sich für Persönlichkeitsmerkmale wie die Neugier, das Interesse oder die Motivation von Lernenden. Entsprechende Merkmale lauten dann ,zeigt mathematische Neugier“ oder „Lösungsweg zeigt Selbstbewusstsein“. Die zweite Frage zielt 
auf die individuellen Vorstellungen und Denkweisen einer Person, die sie bei ihrer mathematischen Auseinandersetzung leiten. Sie führt zu Merkmalen wie ,anschauliches Denken“ oder „hat Grundvorstellung: Log ist Länge der Zahl“. Merkmale wie „Reflexion des eigenen Vorgehens“ und „stellt sich zusätzliche Fragen“ ergeben sich aus dem Blick auf metakognitive und selbstreflexive Prozesse, die genutzt werden, um die Aufgabenbearbeitung voranzutreiben.

\subsubsection{Personal-wertende Perspektive}

Aussagen über eine Person lassen sich nicht nur beschreiben, sie lassen sich auch werten. Aus der personal-wertenden Perspektive stellen sich dann Fragen wie:

Wie nützlich ist der Schülerin oder dem Lernenden ihr oder sein Wissen und Können?

Wo hat sie bzw. er Schwierigkeiten?

Diese Frage zur Nützlichkeit des Wissens und Könnens einer Person führte im Fall unserer befragten Lehramtstudierenden zu Merkmalen wie „kann 2-dim. Fall in der 3-dim. Zeichnung erkennen“ oder „Mühe mit räumlicher Vorstellung“.

\subsection{Quantitative Ergebnisse: Häufigkeiten und Verteilungen}

$\mathrm{Zu}$ Anfang des Semesters waren von den befragten Lehrpersonen 104 Merkmale, in der Mitte des Semesters noch einmal 104 Merkmale und am Ende des Semesters noch 92 Merkmale genannt worden (die wenigen Mehrfachnennungen wurden auch mehrfach gezählt). Da die meisten Grids vier und mehr Gegensatzpaare enthalten, lagen uns zum Schluss der Datenerhebung insgesamt genau 300 Merkmale vor.

In Tabelle 2 sind die absoluten Häufigkeiten der Merkmale jeder Perspektive über die drei Erhebungszeitpunkte dargestellt. Ihr kann entnommen werden, dass jede der sechs Perspektiven zu jedem Erhebungszeitpunkt mindestens einmal eingenommen wurde, auch schon beim ersten Mal. Aus ihr kann aber auch abgelesen werden, dass die inhaltlichbeschreibende Perspektive sowohl zu Beginn als auch in der Mitte des Semesters am häufigsten eingenommen wurde, da dann am meisten entsprechende Merkmale genannt wurden (39- bzw. 42-mal).

\begin{tabular}{l|l:l|}
\hline Beschreibend & Wertend \\
\hline
\end{tabular}




\begin{tabular}{|c|c|c|c|c|c|c|c|c|}
\hline 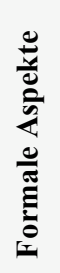 & $\begin{array}{l}\text { Formal-beschreibende } \\
\text { Perspektive: } \\
\text { Graphische Darstellung } \\
\text { Lösungsherleitung }\end{array}$ & $\begin{array}{c}15 \\
5\end{array}$ & $\begin{array}{l}10 \\
0\end{array}$ & $\begin{array}{l}4 \\
5 \\
5\end{array}$ & $\begin{array}{l}\text { Formal-wertende } \\
\text { Perspektive: } \\
\text { Korrektheit } \\
\text { Ästhetik } \\
\text { Verständlichkeit }\end{array}$ & $\begin{array}{c}8 \\
12 \\
9\end{array}$ & $\begin{array}{l}6 \\
4 \\
4\end{array}$ & $\begin{array}{l}1 \\
0 \\
0\end{array}$ \\
\hline 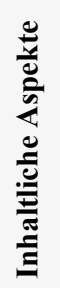 & $\begin{array}{l}\text { Inhaltlich-beschreibende } \\
\quad \text { Perspektive: } \\
\text { Muster und Strukturen } \\
\text { Vorgehensweisen u. Strate- } \\
\quad \text { gien }\end{array}$ & 10 & 42 & 23 & $\begin{array}{l}\text { Inhaltlich-wertende } \\
\text { Perspektive: } \\
\text { Korrektheit } \\
\text { Substanz und Eleganz } \\
\text { Nutzung }\end{array}$ & $\begin{array}{l}0 \\
4 \\
0\end{array}$ & $\begin{array}{l}4 \\
6 \\
2\end{array}$ & $\begin{array}{l}2 \\
4 \\
2\end{array}$ \\
\hline 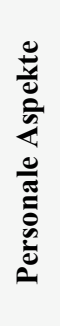 & $\begin{array}{l}\text { Personal-beschreibende } \\
\text { Perspektive: } \\
\text { Persönlichkeitsmerkmale } \\
\text { Vorstellungen u. Denk- } \\
\text { weisen } \\
\text { Metakognitive Prozesse }\end{array}$ & $\begin{array}{l}4 \\
2\end{array}$ & $\begin{array}{l}2 \\
8\end{array}$ & 4 & $\begin{array}{l}\text { Personal-wertende } \\
\text { Perspektive: } \\
\text { Nützlichkeit des Wissens } \\
\text { und Könnens }\end{array}$ & 1 & 12 & 19 \\
\hline
\end{tabular}

Tab. 2: Absolute Häufigkeiten der Merkmale pro Perspektive und pro Erhebungszeitpunkt

Der leichteren Lesbarkeit wegen werden die Anzahlen auch als relative Häufigkeiten dargestellt. So wird sichtbar, dass das Verhältnis von beschreibenden zu wertenden Perspektiven im Verlauf des ganzen Semesters stabil war und bei etwa 2:1 lag (Abb. 4). Eine Untersuchung der einzelnen Perspektiven (Abb. 5 und 6) zeigt, dass ihre Anteile hingegen nicht stabil blieben. So nahm die formal-beschreibende Perspektive von der ersten zur zweiten Erhebung ab. Zwischen der zweiten und dritten Erhebung nahm die inhaltlichbeschreibende Perspektive ab, dafür wurde die personal-beschreibende Perspektive öfter eingenommen. Noch stärker haben sich die verschiedenen wertenden Perspektiven im Verlauf des Semesters verändert. Während die formal-wertende Perspektive bei der ersten Erhebung fast ausschließlich und dann immer weniger oft eingenommen wurde, wurde die personalwertende Perspektive von Erhebung zu Erhebung häufiger eingenommen. 


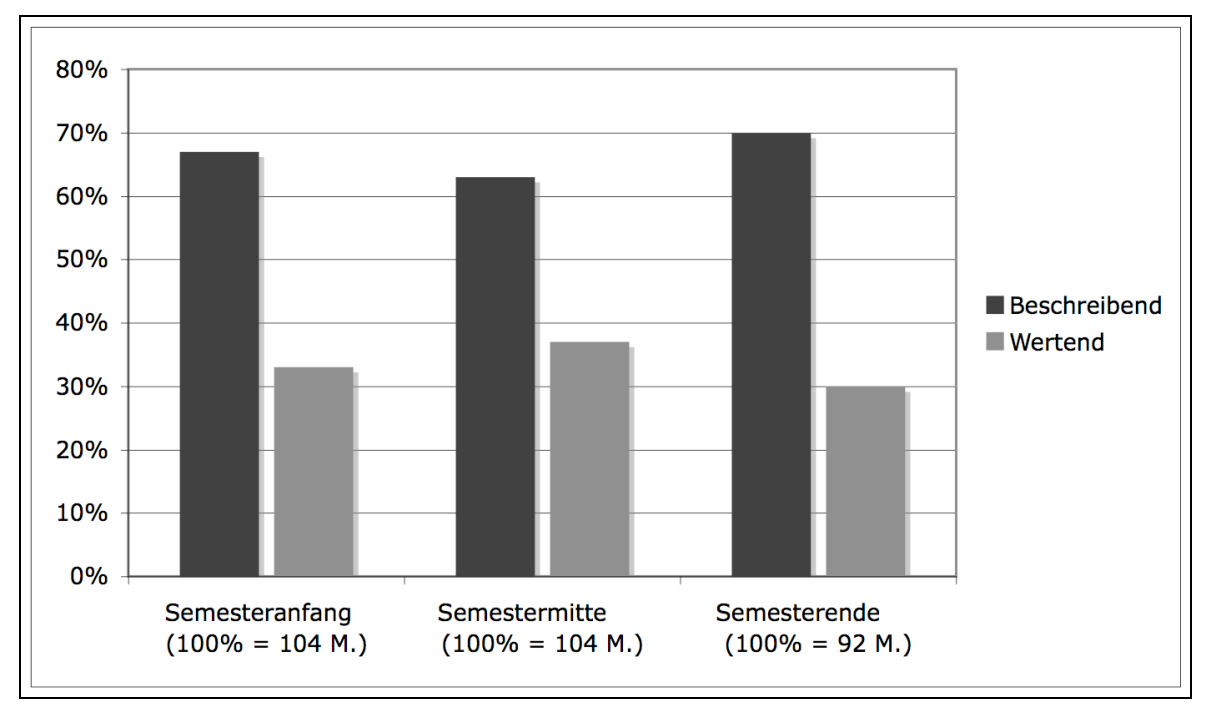

Abb. 4: Anteile der beschreibenden und wertenden Perspektiven im Verlauf des Semesters (M. steht für Merkmale)

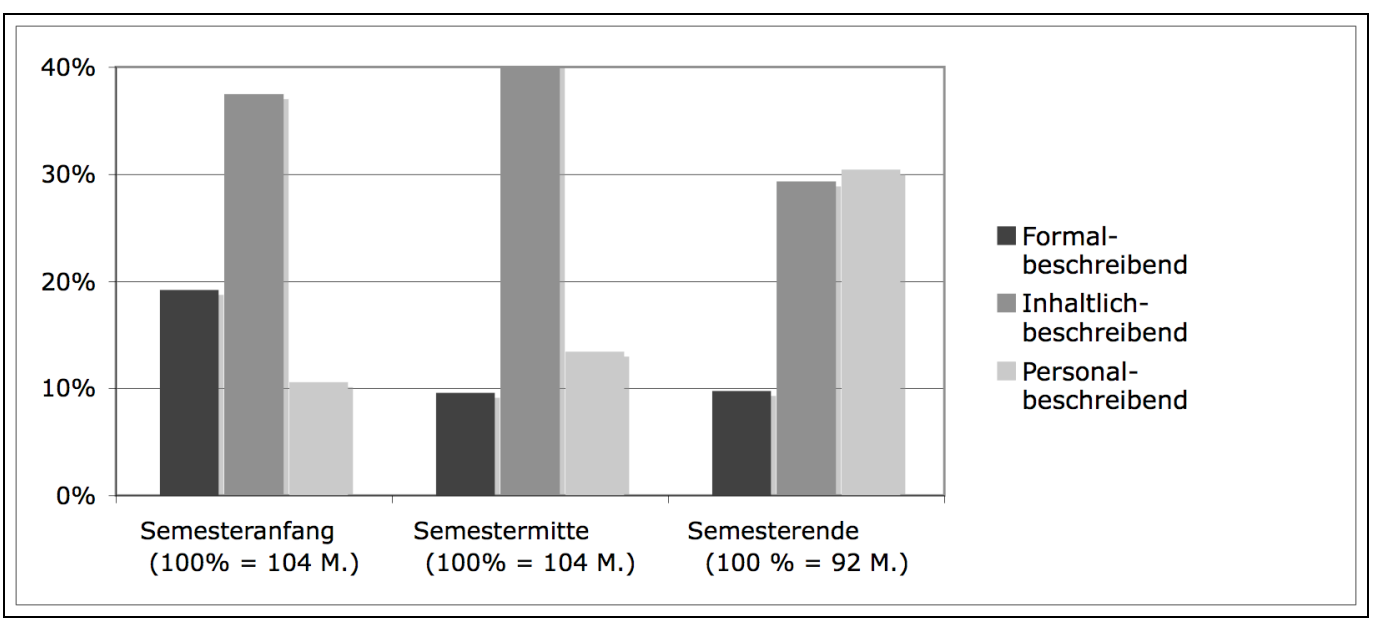

Abb. 5: Anteile der beschreibenden Perspektiven im Verlauf des Semesters

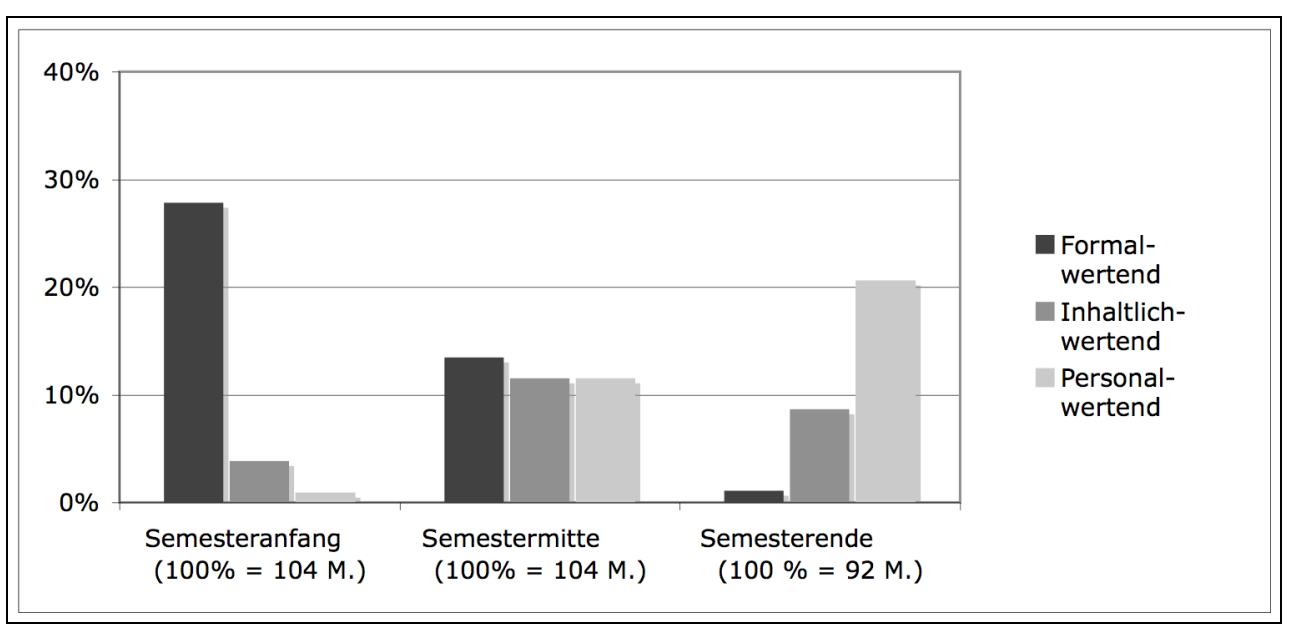

Abb. 6: Anteile der wertenden Perspektiven im Verlauf des Semesters

((An den Setzer: 1. Bitte Abb. 4, 5 und 6 so formatieren, dass sie auf EINER einzigen Seite Platz haben sowie die horizontalen Bereiche der drei Abb. senkrecht unter einander stehen und gleich lang sind.)) 


\section{Diskussion}

Diese Studie zeigt, wie zwölf Schweizer Lehrpersonen, die für den Mathematikunterricht der Sekundarstufe 2 aus- und weitergebildet wurden, die schriftlich protokollierten Aufgabenbearbeitungen von Schülerinnen und Schülern lasen. Dazu wurde mit Hilfe von Repertory Grids rekonstruiert, aus welchen Perspektiven sie auf solche Schülerprotokolle schauten. Die eingenommenen Perspektiven sind überraschend vielfältig. So konnten aus insgesamt 300 Merkmalen sechs verschiedene Perspektiven rekonstruiert und beschrieben werden: eine formal-beschreibende, eine formal-wertende, eine inhaltlich-beschreibende, eine inhaltlich-wertende, eine personal-beschreibende und eine personal-wertende Perspektive. In diesem Sinne ordnet das Kategoriensystem in Tabelle 1 die Vielfalt der tatsächlich eingenommenen Leseperspektiven der befragten Lehrerinnen und Lehrer. Da die vorgestellte Studie explorativen Charakter hat, ist ein erster Schritt getan. Folgestudien müssen jedoch zeigen, welche weiteren Perspektiven und unter welchen Bedingungen welche Perspektiven beim Lesen von Schülertexten eingenommen werden, um die hier vorgestellten Befunde zu ergänzen und zu differenzieren.

Perspektiven können gewechselt werden. Genau dies haben die Versuchspersonen bereits während der Lektüre getan, einfach weil das Ausfüllen eines Repertory Grids dies erfordert (Abb. 3). Darüber hinaus verschoben sich im Verlauf des Semesters ihre Präferenzen für bestimmte Perspektiven (Abb. 5 und 6). Worauf diese Änderung zurückzuführen ist, kann aufgrund der vorliegenden Daten nicht beantwortet werden. Ob es sich dabei um den Einfluss der Lehrveranstaltung, die wiederholte Arbeit mit Repertory Grids oder gar um soziale Erwünschtheit handelt, muss an dieser Stelle unklar bleiben. Dazu wie auch zur Frage der Stabilität dieser Änderungen sind Folgestudien nötig.

Im Folgenden werden die sechs Perspektiven kurz diskutiert. Dann wird von vorläufigen Erfahrungen ihres Einsatzes in der Lehreraus- und -weiterbildung berichtet.

\subsection{Die sechs Perspektiven und ihre didaktische Relevanz}

Wie das Kategoriensystem (Tab. 1) zeigt, lassen sich die rekonstruierten Perspektiven unterschiedlich gliedern. So sind entweder drei Perspektiven beschreibend und drei wertend, oder aber zwei Perspektiven sind formal, zwei inhaltlich und zwei personal. Mit den nun folgenden Ausführungen soll gezeigt werden, dass die rekonstruierten Perspektiven nicht nur empirisch belegbar, sondern auch didaktisch relevant sind. 


\subsubsection{Beschreibende und wertende Perspektiven}

Die erste Unterscheidung in drei beschreibende und drei wertende Perspektiven spiegelt ganz allgemein zwei disjunkte Sichtweisen, unter denen ein Sachverhalt betrachtet werden kann, wider. Der Wissenschaftstheoriker Hoyningen-Huene (1987) trennt beispielsweise zwischen einer faktischen („factual“) und einer normativen (,normative“) Perspektive. Die Einführung dieser beiden Perspektiven geschieht vor dem Hintergrund der in der Wissenschaftstheorie bis heute kontrovers geführten Diskussion um die Unterscheidung eines Entdeckungs- und eines Rechtfertigungskontexts. Nach der Darstellung der wesentlichen Argumente für und gegen die Unterscheidung dieser beiden Kontexte hält Hoyningen-Huene lapidar fest:

„It is striking that none of the attacks on the context distinction has been directed against the distinction between the factual and the normative. [...] But this difference seems to be the core of the context distinction as intended by its proponents." (Hoyningen-Huene 1987, S. 511)

Aus einer faktischen Perspektive heraus zielt man auf eine möglichst genaue Beschreibung, aus einer normativen Perspektive heraus findet eine Beurteilung, Auswertung und Einschätzung eines Sachverhalts statt. Mit anderen Worten entspricht Hoyningen-Huenes „factual“ unseren beschreibenden Perspektiven, und sein ,normative“ entspricht den wertenden Perspektiven.

In der Mathematik-Didaktik wird diese Unterscheidung insbesondere bei der Diskussion von Verstehensprozessen einzelner Schülerinnen und Schüler sichtbar. Beispielsweise trennen Maier und Steinbring (1998) zwischen der Beschreibung und der Evaluation des Verstehens, wenn sie präzisieren:

„Genauer gesagt wird zuerst gefragt, was und wie der einzelne Schüler verstanden hat, und erst dann, wie weit dieses sein Verstehen gewissen empirischen Vorgaben bzw. impliziten und expliziten Normen gerecht wird.“ (Maier u. Steinbring 1998, S. 297) Maier und Steinbring trennen die Beschreibung und die Wertung (Evaluation) aber auch zeitlich, denn die Beschreibung geht der Wertung grundsätzlich voran. Damit ist zu vermuten, dass die Lehrpersonen auch für die Rekonstruktion individueller Denkwege zuerst eine der drei beschreibenden Perspektiven einnehmen müssen, bevor sie diese Denkwege werten können. Erst nachdem man verstanden hat, wie jemand in seiner Aufgabenbearbeitung vorgegangen ist, kann entschieden werden, unter welchen wertenden Perspektiven sich die Lektüre lohnen könnte. 


\subsubsection{Formale, inhaltiche und personale Perspektiven}

Die Perspektiven können aber auch ganz anders gegliedert werden. So richtet sich gemäß den Zeilen des Kategoriensystems jede Perspektive entweder auf formale oder auf inhaltliche oder auf personale Aspekte eines Texts. Welche für den Unterricht relevanten Bereiche werden damit erfasst und welche nicht?

In der erwähnten Studie von Maier (2002) werden Möglichkeiten genannt, wie Lehrpersonen Schülerprotokolle interpretieren können. Schülerprotokolle angemessen interpretieren zu können, ist für Maier die Voraussetzung, dass sich die Lehrperson über das Wissen und Können der Lernenden informieren kann. Solange man ein deskriptives Verstehensmodell voraussetzt, kann sich die Aufmerksamkeit der Lehrperson auf „modale“ sowie „mentale Aspekte“ beziehen (vgl. auch Maier u. Steinbring 1998). Die modalen Aspekte von Maier entsprechen unserer formal-beschreibenden Perspektive, und seine mentalen Aspekte entsprechen unserer inhaltlich-beschreibenden Perspektive. Erst wenn bei der Interpretation auch nicht-deskriptiv vorgegangen wird, können nach Maier normative Aspekte einbezogen werden, aber auch solche, die wir bei unserem Kategoriensystem unter die personalen Aspekte subsumiert haben. Insgesamt zeigt sich, dass unsere Perspektiven die auch für Maier möglichen Interpretationen von Schülerprotokollen mit umfassen.

Schülerprodukte lassen sich für diagnostische Zwecke heranziehen, also für die Frage, was Schülerinnen und Schüler können, um dann zu entscheiden, wie sie in ihrem Lernprozess unterstützt werden können. So geben Hußmann, Leuders und Prediger (2007, S. 3) eine Liste von Könnens- und Wissensaspekten an, die für Lernprozesse wichtig sind und auf die sich eine Diagnose folglich beziehen kann. Darunter fallen „mathematikspezifische“ Aspekte wie Verfahren, inhaltliche Vorstellungen, Begriffe, Regeln sowie prozessbezogene Kompetenzen, aber auch Kreativität, Denkstile und Einstellungen. Unter „überfachlichen Aspekten“ sind metakognitive Kompetenzen, Arbeitsweisen, motivationale und affektive Aspekte sowie soziale Kompetenzen subsumiert. Diese Aspekte sind auch mit unserem Begriff der Perspektiven „in den Blick“ genommen. Der wesentliche Unterschied zwischen der Liste von Hußmann, Leuders und Prediger und unserem Kategoriensystem bzw. den rekonstruierten Perspektiven liegt weniger im abgesteckten Bereich als vielmehr in der Art und Weise der Ausdifferenzierung. 
Aufgrund der auf Exploration ausgerichteten Forschungsfrage haben wir ein methodisches Design gewählt, das möglichst keine Leseperspektive vorgibt. Dies führte dazu, dass die Schülertexte nur vereinzelt in Hinblick auf den Unterricht angeschaut wurden. Würde der Blick der befragten Lehrpersonen stärker auf die konkrete Unterrichtspraxis gelenkt, würden entsprechende unterrichtspraktische Merkmale vermutlich häufiger genannt. Die COACTIVStudie beispielsweise fokussiert auf den Zusammenhang von Lehrerwissen und Unterrichtsqualität (Krauss et al. 2008), entsprechend konzeptualisiert sie fachdidaktisches Wissen von Lehrpersonen als das Wissen ,über das Verständlichmachen von mathematischen Inhalten“ (also wie Inhalte erklärt und dargestellt werden), „über mathematikbezogene Schülerkognitionen“ (zum Beispiel typische Schülerschwierigkeiten) und „über das kognitive Potential von Mathematikaufgaben“ (etwa strukturelle Unterschiede verschiedener Lösungswege) (ebd., S. 234). Besonders die erste, aber auch die dritte Komponente beziehen sich auf das Unterrichtsgeschehen. Als solche liegen sie nicht im Kernbereich unseres Kategoriensystems. Einzig die wenigen Merkmale der inhaltlich-wertenden Perspektive, die sich auf die Frage nach der Nutzung beziehen, verweisen explizit darauf, dass auch in unserer Studie Schülerprotokolle punktuell im Hinblick auf den weiteren Unterricht gelesen wurden. Andere direkt auf den Unterricht bezogene Perspektiven ließen sich nicht rekonstruieren.

In einer Folgeuntersuchung wollen wir deshalb die Leseperspektive entsprechend lenken. In ihr sollen die Lehrpersonen geeignete Textabschnitte aus Schülerprotokollen auswählen und daraus Unterrichtsmaterial für den anschließenden Unterricht entwickeln. So wird zum einen das erkundete Terrain, das die vorliegende Studie absteckt, für das Unterrichtshandeln produktiv gemacht. Zum anderen wird ein weiterer Schritt in Richtung einer Nutzung von Schülerangeboten getan (siehe Abschnitt 1).

\subsection{Mehrperspektivität und Lehrerbildung}

In diesem Abschnitt wird zuerst ein quantitatives Ergebnis diskutiert, dass nämlich die befragten Lehrpersonen in der Lage waren, unterschiedliche Leseperspektiven einzunehmen (Abschnitt 5.2). Anschließend wird angedacht, welche Konsequenzen diese Studie für die Aus- und Weiterbildung von Lehrpersonen haben könnte.

\subsubsection{Lehrpersonen können durchaus unterschiedliche Perspektiven einnehmen}

Lehrpersonen befinden sich in ihrem Unterrichtsalltag sehr oft in Beurteilungssituationen, nicht zuletzt weil es in der Schule immer auch um die Frage der Einstufung von Personen 
geht (Sacher 2009). So ist es wenig verwunderlich, dass Lehrpersonen im Laufe ihrer beruflichen Sozialisierung lernen, dass auch Schülerprotokolle unter einer formal-wertenden Perspektive zu lesen sind. Entsprechend stellt Maier (2002, S. 145) eine ausgeprägte Neigung der Lehrpersonen fest, die Eigenproduktionen von Schülerinnen und Schülern unter einer formal-wertenden Perspektive zu lesen:

„Bei interpretierenden Lehrern zeigt sich eine deutliche Neigung, an die Eigenproduktionen eines Schülers mit ganz bestimmten Erwartungen heranzugehen. Sie messen diese gleichsam an ihrer Einschätzung dessen, was mathematisch richtig und korrekt bzw. falsch oder unerlaubt ist, oder daran, wie sie den betreffenden Sachverhalt den Schülern im vorausgehenden Unterricht vorgestellt haben. Eine solch normative, im Wesentlichen an den Kriterien ,vollständig' oder ,unvollständig‘ bzw. ,richtig‘ und ,falsch“ orientierte Interpretation verstellt den Blick dafür, welche Art von Wissen und Verstehen der einzelne Schüler tatsächlich konstruiert hat.“

Im Widerspruch dazu weisen unsere Ergebnisse darauf hin, dass Lehrpersonen unter gewissen entfremdenden Bedingungen durchaus in der Lage sein können, auch andere Perspektiven einzunehmen. So stammen die Texte von (den Versuchspersonen) unbekannten Schülerinnen und Schülern und mussten nicht im Hinblick auf den eigenen Unterricht gelesen werden. Darüber hinaus waren die Regeln der Repertory Grids zu befolgen und immer mehrere Merkmalspaare zu nennen. Wer einen Schülertext unter einer formal-wertenden Perspektive liest, erschöpft sich bald in einigen wenigen Merkmalen. Um fünf Merkmalspaare nennen zu können, bleibt nichts anderes übrig, als anders auf die Aufgabenbearbeitung zu schauen und etwas anderes wahrzunehmen, etwa weiterführende Ideen oder dass jemand sein Vorgehen reflektiert hat.

Daher ist das Ergebnis unserer Studie auch dahingehend zu interpretieren, dass Lehrpersonen durchaus mehr als eine Perspektive auf Schülertexte einnehmen können. Mindestens bei den von uns befragten Lehrpersonen und unter den gegebenen Bedingungen war dies der Fall.

\subsubsection{Ausbildung eines mehrperspektivischen Umgangs mit Schülertexten}

Es scheint uns plausibel, dass Lehrpersonen die Aufgabenbearbeitungen von Lernenden nur dann adäquat interpretieren - und damit auch für den Unterricht nutzen - können, wenn sie sie aus mehreren Perspektiven lesen können. Allerdings reichen mehrere Perspektiven alleine nicht aus, wenn sie nicht auch inhaltlich stimmig und konsistent gewichtet sind. 
Wie man Lehrpersonen zu einem mehrperspektivischen Umgang mit Schülertexten befähigt, kann an dieser Stelle nicht abschließend beantwortet werden. Ob es besser ist, Lehrpersonen zur Anwendung des Kategoriensystems bzw. der sechs Perspektiven zu trainieren oder sie mittels Repertory Grids zur Einnahme unterschiedlicher Perspektiven anzuhalten, kann aus der vorliegenden Studie nicht abgeleitet werden. Dennoch möchten wir unseren Bericht mit einigen Beobachtungen und Erfahrungen abschließen.

Sollen Lehrpersonen in ihrer Aus- oder Weiterbildung den praktischen Gebrauch des Kategoriensystems kennen lernen, könnte so vorgegangen werden, dass die Perspektiven ähnlich wie in Abschnitt 5.1 anhand von Leitfragen und Ankerbeispielen erklärt werden. Wenn man dann an einen Text spezifische Fragen (beispielsweise: „Wie wurde der Text graphisch gestaltet und dargestellt? Wie wurde die Herleitung der Lösung festgehalten?“) stellt, nimmt man eine bestimmte (hier: formal-beschreibende) Leseperspektive ein. Die Ankerbeispiele unserer Lehrpersonen können dann zur Verdeutlichung der jeweiligen Perspektive helfen. Wie gut dies gelingt, kann nicht prognostiziert werden, wenn auch die Ankerbeispiele, die ja in der Sprache von Lehrpersonen verfasst sind, begründeten Anlass zur Hoffnung geben. Vielleicht würde die eine oder andere Perspektive noch besser verständlich, wenn den Lehrpersonen stattdessen (fiktive) beispielhafte Merkmale vorgelegt würden.

Die Ausbildung eines mehrperspektivischen Umgangs mit Schülertexten könnte aber auch durch Repertory Grids erreicht werden. So hat diese Methode in unserer Untersuchung vermutlich nicht nur zur Einnahme unterschiedlicher Perspektiven, sondern gerade auch zur Ausbildung eines entsprechenden Umgangs wesentlich beigetragen. Diese Hypothese wird durch mehrere Beobachtungen gestützt. So hat sich dank der Repertory Grids der Umgang mit Schülertexten erst einmal verlangsamt. Darüber hinaus haben sich die Lehrpersonen mehrfach mit jedem einzelnen Text auseinandergesetzt und so immer wieder auch Qualitäten feststellen können. In der Folge trugen die Arbeit mit sowie der Vergleich von Repertory Grids mindestens zur „Entwicklung von Bewusstheit“ (Selter 1995) bei, der Bewusstheit also, dass die Leseperspektive den Umgang mit Schülertexten wesentlich mitbestimmt.

Derzeit versuchen wir bei Lehramtsstudierenden der Vor- und Grundschule erste Schritte eines mehrperspektivischen Umgangs mit Schülertexten zu initiieren. Dazu geben die Studierenden selbst einmal monatlich die Bearbeitung einer mathematischen Aufgabe ab. 
Anschließend erhalten sie alle Protokolle der Gruppe und haben sie unter zwei

Gesichtspunkten zu sichten: Erstens sind zwei möglichst „verschiedene“ und zweitens eine „interessante“ Aufgabenbearbeitung herauszugreifen und zu diskutieren. Auch wenn dieses Vorgehen einfacher ist als die Methode der Repertory Grids, lehnt es sich unverkennbar daran an. Über seine Folgen wird an anderer Stelle zu berichten sein.

Damit ist nicht geklärt, wie Lehrpersonen darin unterstützt werden können, mehrere und adäquate Leseperspektiven im Umgang mit Schülerprodukten zu entwickeln. Weiterführende Studien müssen zeigen, ob sich unsere Ergebnisse und Beobachtungen theoretisch wie praktisch fundieren lassen oder nicht.

Danksagung Ein herzlicher Dank geht an die Gutachterinnen und Gutachter für ihre konstruktiven und hilfreichen Hinweise.

\section{Literatur}

Atteslander, P. (2010). Methoden der empirischen Sozialforschung. Berlin: Erich Schmidt.

Ballstaedt, S. P., Mandl, H., Schnotz, W., Tergan, S. O. (1981). Texte verstehen, Texte gestalten. München: Urban \& Schwarzenberg.

Badr Goetz, N. (2007). Das Dialogische Lernmodell. München: Meidenbauer.

Borromeo Ferri, R. (2004). Mathematische Denkstile - Ergebnisse einer empirischen Studie. Hildesheim: Franzbecker. Bruder, R., Lengnink, K., Prediger, S. (2003). Wie denken Lehramtsstudierende über Mathematikaufgaben? mathematica didactica, 26 (1), 63-85.

Chi, M., Feltovich, P., Glaser, R. (1981). Categorization and representation of physics problems by experts and novices.

Cognitive Science, 5, 121-152.

Christmann, U. (2004). Lesen. In: R. Mangold, P. Vorderer, G. Bente (Hrsg.), Lehrbuch der Medienpsychologie (S. 419-

442). Göttingen: Hogrefe.

Dahlke, E. (1978). Krutetskii - The Psychology of Mathematical Abilities in Schoolchildren. Zentralblatt für Didaktik der Mathematik, 10 (2), 84-86.

Deller, H., Gebauer P., Zinn, J. (2000). Algebra (Aufgaben, Band 2). Zürich: Orell Füssli.

Dörfler, W. (2008). Mathematical Reasoning: Mental Activity or Practice with Diagrams. In: Niss, M. (Hrsg.), Proceedings of the Tenth International Congress on Mathematical Education. Roskilde: IMFUFA, Roskilde University.

Drüke-Noe, C. (2009). Mathematische Texte - auch in Klassenarbeiten. mathematik lehren, Heft 156, 52-57.

Fend, H. (1998). Qualität im Bildungswesen - Schulforschung zu Systembedingungen, Schulprofilen und Lehrerleistungen.

Weinheim, München: Juventa.

Fischer, A. (2006). Der Einsatz von Zeichen als Werkzeuge zur mentalen Konstruktion abstrakter Objekte. Journal für Mathematikdidaktik, 27 (3/4), 180-199.

Fischler, H. (2000). Über den Einfluss von Unterrichtserfahrungen auf die Vorstellungen vom Lehren und Lernen bei Lehrerstudenten der Physik. Zeitschrift für Didaktik der Naturwissenschaften, 6, 79-95.

Fransella, F., Bell, R., Bannister, D. A. (1977). A Manual for Repertory Grid Technique, London: Academic Press. 
Grzesik, J. (1990). Textverstehen lernen und lehren. Geistige Operationen im Prozess des Textverstehens und typische Methoden für die Schulung zum kompetenten Leser. Stuttgart: Klett.

Hafner, T. \& vom Hofe, R. (2008). Aufgaben analysieren und Schülervorstellungen erkennen. mathematik lehren, Heft 150, $14-19$.

Heinemann, M. \& Viehweger, D. (1991). Textlinguistik. Tübingen: Max Niemeyer.

Helmke, A. (2006). Was wissen wir über guten Unterricht? Über die Notwendigkeit einer Rückbesinnung auf den Unterricht als dem „Kerngeschäft“ der Schule. Pädagogik, 58 (2), 42-45.

Helmke, A. (2009). Unterrichtsqualität und Lehrerprofessionalität. Diagnose, Evaluation und Verbesserung des Unterrichts. Seelze: Kallmeyer.

Hill, H. C., Loewenberg Ball, D., Schilling, S. G. (2008). Unpacking Pedagogical Content Knowledge: Conceptualizing and Measuring Teachers' Topic-Specific Knowledge of Students. Journal for Research in Mathematics Education, 39 (4), $372-$ 400.

Hiskonen, K. (1999). A good pupil's belief about mathematics learning assessed by repertory grid methodology. In: O. Zaslavsksy (Hrsg.), Proceedings of the 23rd Conference of the International Group for the Psychology of Mathematics Education 3 (S. 97-104). Haifa: Israel Institute of Technology.

vom Hofe, R. (1995). Grundvorstellungen mathematischer Inhalte. Heidelberg: Spektrum.

vom Hofe, R. (2003). Grundbildung durch Grundvorstellungen. mathematik lehren, Heft 118, 4-8.

Holzäpfel, L., Glogger, I., Schwonke, R., Nückles, M., Renkl, A. (2009). Lernstrategien beim Schreiben: Neue Anregungen für den Umgang mit dem Lerntagebuch. mathematik lehren, Heft 153, 16-21.

Hörmann, H. (1980). Der Vorgang des Verstehens. In: W. Kühlwein \& A. Raasch (Hrsg.), Sprache und Verstehen.

Kongressberichte der 10. Jahrestagung der Gesellschaft für Angewandte Linguistik GAL e. V., Mainz 1979 (S. 17-29).

Tübingen: Gunter Narr.

Hoyningen-Huene, P. (1987). Context of Discovery and Context of Justification. Studies in History and Philosophy of Science, 18 (4), 501-515.

Hußmann, S. (2003). Lerntagebücher. In: T. Leuders (Hrsg.), Mathematik-Didaktik: Praxishandbuch für die Sekundarstufe I und II (S. 75-92). Berlin: Cornelsen Scriptor.

Hußmann, S., Leuders, T., Prediger, S. (2007). Schülerleistungen verstehen - Diagnose im Alltag. Praxis der Mathematik in der Schule, Heft 15, 1-8.

Jordan, A. \& vom Hofe, R. (2008). Diagnose von Schülerleistungen: „Schlüssel” zur individuellen Förderung. mathematik lehren, Heft 150, 4-12.

Jungwirth, H., Steinbring, H., Voigt, J., Wollring, B. (2001). Interpretative classroom research in teacher education. In: H.-G. Weigand et al. (Hrsg.), Selected papers from the Annual Conference of Didactics of Mathematics 1996 (S. 49-56).

Hildesheim: Franzbecker.

Kaakinen, J. K. \& Hyönä, J. (2005). Perspective Effects on Expository Text Comprehension: Evidence from Think-Aloud Protocols, Eyetracking, and Recall. Discourse Processes, 40 (3), 239-257.

Kaune, C. (2007). „Der denkt irgendwie anders als ich“- Spuren kognitiver Strukturen in Schüleräußerungen. Praxis der Mathematik in der Schule, Heft 15, 23-30.

Kelly, G. A. (1955). The psychology of personal constructs. New York: Norton.

Krauss, S., Neubrand, M., Blum, W., Baumert, J., Brunner, M., Kunter, M., Jordan, A. (2008). Die Untersuchung des professionellen Wissens deutscher Mathematik-Lehrerinnen und -Lehrer im Rahmen der COACTIV-Studie. Journal für Mathematik-Didaktik, 29 (3/4), 223-258.

Krutetskii, V. A. (1976). The Psychology of Mathematical Abilities in Schoolchildren. Chicago \& London: The University of Chicago Press.

Maier, H. (2002). Zur Interpretation textlicher Eigenproduktionen von Schülern. In: A. Peter-Koop (Hrsg.), Das besondere Kind im Mathematikunterricht der Grundschule (S. 141-154). Offenburg: Mildenberger. 
Maier, H. \& Schweiger, F. (1999): Mathematik und Sprache - Zum Verstehen und Verwenden von Fachsprache im Mathematikunterricht. Wien: öbv \& hpt.

Maier, H. \& Steinbring H. (1998). Begriffsbildung im alltäglichen Mathematikunterricht. Vergleich zweier Theorieansätze zur Analyse von Verstehensprozessen. Journal für Mathematik-Didaktik, 19 (4), 292-329.

Mayring, P. (2008a). Qualitative Inhaltsanalyse - Grundlage und Techniken. Weinheim \& Basel: Beltz.

Mayring, P. (2008b). Neuere Entwicklungen in der qualitativen Forschung und der qualitativen Inhaltsanalyse. In: P. Mayring \& M. Gläser-Zakuda (Hrsg.), Die Praxis der Qualitativen Inhaltsanalyse (S. 7-19). Weinheim \& Basel: Beltz. Moskal, B. M. \& Magone, M. E. (2000). Making Sense of what Students Know: Examing the Referents, Relationships and Modes Students Displayed in Response to a Decimal Task. Educationals Studies in Mathematics, 43, 313-335.

Pichert, J. W. \& Anderson, R. C. (1977). Taking Different Perspectives on a Story. Journal of Educational Psychology, 69 (4), 309-315.

Prediger, S. (2010). How to develop mathematics for teaching and for understanding. The case of meanings of the equal sign. Journal of Mathematics Teacher Education, 13 (1), 73-93.

Rüede, C. \& Weber, C. (2009). Analyse von Schülerprotokollen mit Repertory Grids. Download unter http://www.fhnw.ch/personen/christof-weber/dateien/RepGrids_2009.pdf (7,0 MB)

Ruf, U. \& Gallin, P. (1998). Dialogisches Lernen in Sprache und Mathematik, Band I und II. Seelze: Kallmeyer.

Ruf, U. (2008). Das Dialogische Lernmodell vor dem Hintergrund wissenschaftlicher Theorien und Befunde. In: U. Ruf, S. Keller, F. Winter (Hrsg.), Besser lernen im Dialog (S. 233-270). Seelze: Friedrich.

Ruf, U. \& Winter, F. (2006). Qualitäten finden. Der Blick auf Defizite hilft nicht weiter. In G. Becker, M. Horstkemper, E. Risse, L. Stäudel, R. Werning, F. Winter (Hrsg.), Diagnostizieren und Fördern. Stärken entdecken - Können entwickeln . Friedrich Jahresheft 24 (S. 56-59). Seelze: Friedrich.

Sacher, W. (2009). Leistungen entwickeln, überprüfen und beurteilen. Bad Heilbrunn: Klinkhardt.

Scheer, J. W. (1992). Psychologie der persönlichen Konstrukte und Repertory Grid-Technik. Ein idiographischer Ansatz in klinischer und medizinischer Psychologie. In: L. R. Schmidt (Hrsg.), Psychologische Aspekte medizinischer Maßnahmen. Jahrbuch der medizinischen Psychologie, Bd. 7 (S. 273-290). Berlin: Springer.

Schraw, G. \& Sperling-Dennison, R. (1994). The Effect of Reader Purpose on Interest and Recall. Journal of Reading Behavior, 26 (1), 1-18.

Schwank, I. (1996). Zur Konzeption prädikativer versus funktionaler kognitiver Strukturen und ihrer Anwendung. Zentralblatt für Didaktik der Mathematik, 28 (6), 168-183.

Schwank, I. (2003). Einführung in prädikatives und funktionales Denken. Zentralblatt für Didaktik der Mathematik, 35 (3), 70-78.

Selter, C. (1994). Eigenproduktionen im Arithmetikunterricht der Primarstufe. Wiesbaden: Deutscher Universitätsverlag. Selter, C. (1995). Entwicklung von Bewusstheit als eine zentrale Aufgabe der Grundschullehrerbildung. Journal für Mathematik-Didaktik, $16(1 / 2), 115-144$.

Spangenberg, N. \& Wolff, K. E. (1993). Datenreduktion durch die Formale Begriffsanalyse von Repertory Grids. In: J. W. Scheer \& A. Catina (Hrsg.), Einführung in die Repertory Grid-Technik, Bd. 2 (S. 38-54). Bern: Hans Huber. Spiegel, H. \& Selter, C. (2007). Kinder \& Mathematik. Was Erwachsene wissen sollten. Seelze: Kallmeyer. Spitta, G. U. (1999). Aufsatzbeurteilung heute: Der Wechsel vom Defizitblick zur Könnensperspektive (I). Grundschulunterricht, 46 (4), 23-27.

Thomas, G. \& James, D. (2006). Reinventing grounded theory: Some questions about theory, ground and discovery. British Educational Journal, 32, 767-795.

Williams, S. R., Khisty, L. L. \& Pack, M. (1997). Teachers' beliefs about mathematics as assessed with repertory grid methodology. In: J. A. Dossey et al. (Hrsg.), Proceedings of the 19th Annual Meeting of the North American Chapter of the International Group for the Psychology of Mathematics Education, 2 (S. 453-458). Columbus, OH: ERIC Clearinghouse. 
Wollring, B. (1998). Beispiele zu raumgeometrischen Eigenproduktionen in Zeichnungen von Grundschulkindern. In: H. Becher, H. R. Bennack, E. Jürgens (Hrsg.), Taschenbuch Grundschule (S. 126-140). Baltmannsweiler: Schneider Hohengehren. 


\section{Anhang}

\section{Legende Abbildungen:}

Abb. 1: Das Angebot-Nutzungs-Modell von Unterricht (vereinfacht, nach Fend und Helmke) und seine Erweiterung (nach Badr Goetz und Ruf)

Abb. 2: Offener Auftrag an die Schülerinnen und Schüler

Abb. 3: Ausgefülltes Repertory Grid von Nicole Müller (um $-90^{\circ}$ verdreht abgebildet)

Abb. 4: Anteile der beschreibenden und wertenden Perspektiven im Verlauf des Semesters (M. steht für Merkmale)

Abb. 5: Anteile der beschreibenden Perspektiven im Verlauf des Semesters

Abb. 6: Anteile der wertenden Perspektiven im Verlauf des Semesters

\section{Legende Tabellen:}

Tab. 1: Kategoriensystem mit Ankerbeispielen und Perspektiven

Tab. 2: Absolute Häufigkeiten der Merkmale pro Perspektive und pro Erhebungszeitpunkt 